\title{
Ballistic spin field-effect transistors: Multichannel effects
}

\author{
Jae-Seung Jeong A and Hyun-Woo Lee \\ PCTP and Department of Physics, Pohang University of Science and Technology, Pohang, Kyungbuk 790-784, Korea
}

\begin{abstract}
We study a ballistic spin field-effect transistor (SFET) with special attention to the issue of multichannel effects. The conductance modulation of the SFET as a function of the Rashba spin-orbit coupling strength is numerically examined for the number of channels ranging from a few to close to 100. Even with the ideal spin injector and collector, the conductance modulation ratio, defined as the ratio between the maximum and minimum conductances, decays rapidly and approaches one with the increase of the channel number. It turns out that the decay is considerably faster when the Rashba spin-orbit coupling is larger. Effects of the electronic coherence are also examined in the multichannel regime and it is found that the coherent Fabry-Perot-like interference in the multichannel regime gives rise to a nested peak structure. For a nonideal spin injector/collector structure, which consists of a conventional metallic ferromagnet-thin insulator-2DEG heterostructure, the Rashba-coupling-induced conductance modulation is strongly affected by large resonance peaks that arise from the electron confinement effect of the insulators. Finally scattering effects are briefly addressed and it is found that in the weakly diffusive regime, the positions of the resonance peaks fluctuate, making the conductance modulation signal sample-dependent.

PACS numbers: 72.25.Dc, 85.75.Hh
\end{abstract}

\section{INTRODUCTION}

At an interface of a semiconductor heterostructure, electrons confined to the interface form a twodimensional electron gas (2DEG). The Rashba spin-orbit (RSO) coupling $\frac{1}{1}$ arises in the 2DEG when the interface confinement potential breaks the structural inversion symmetry. It is demonstrated that the strength of the RSO coupling can be modulated ${ }^{2}$ by a gate voltage that affects the asymmetry of the confinement potential. The RSO coupling can be a useful tool for spintronic applications in semiconductors ${ }^{3}$. One of representative examples is the spin field-effect transistor (SFET) proposed by Datta and Das $\underline{4}$, which is based on the spin precession by the RSO coupling within the 2DEG and on the spin preparation and detection by two spin-selective electrodes (injector and collector).

Despite intensive experimental efforts, the SFET has not been realized yet and there are numerous reports addressing practical problems for the realization of the SFET such as the spin injection/detection ${ }^{5,6}$ and the spin relaxation ${ }^{7-\underline{9}}$. In this paper, we focus on the issue of the multichannel effects. While a narrow 2DEG with only one transport channel is an ideal environment for the SFET operation $\underline{10}$, a single-channel system is rather difficult to realize in experiments. For a 2DEG with the Fermi wavelength of $100 \mathrm{~A}$, for instance, the width of the system needs to be smaller than $100 \mathrm{~A}$ for the system to be in the single channel regime. Preparing a 2DEG with its width less than $100 \mathrm{~A}$ is, though not impossible, quite demanding. The experimental difficulty is further enhanced when it is taken into account that for the transistor operation, the system needs to be longer than a certain minimum length for the spin precession angle to be of order $\pi$ at least. For the 2DEG formed at the InGaAs/InAlAs interface, for instance, the minimum length was estimated to be $0.67 \mu \mathrm{m}^{4}$. Taking into account the experimental difficulty in the preparation of a sufficiently long single-channel system, a multichannel system is thus a more practical test ground for the SFET.

Behaviors of a multichannel SFET may be crucially affected by the inter-channel coupling. In Ref $\underline{\underline{\underline{4}}}$, it was argued that effects of the inter-channel coupling will be negligible and a multichannel SFET will behave as a collection of uncoupled single-channel SFETs when a dimensionless number $2 m^{*} \alpha w / \hbar^{2}$ is sufficiently smaller than $2 \pi$, where $w$ is the width of the 2DEG, $m^{*}$ is the effective mass of the electron in the 2DEG, and $\alpha$ is the RSO coupling parameter. In this paper, we examine systematically behaviors of a multichannel SFET with the number of channels from a few to close to 100 and with $\alpha$ from $10^{-12} \mathrm{eVm}$ to $10^{-10} \mathrm{eVm}$, covering most reported values of $\alpha^{2}, \underline{11}-\underline{14}$. For the examined range, the value of $2 m^{*} \alpha w / \hbar^{2}$ varies from approximately 0.03 to 50 , including the range where the inter-channel coupling is not negligible. Our result thus provides a systematic investigation of the multichannel effects. We also examine effects of the electronic coherence in the multichannel regime. For a single-channel SFET, it was recently demonstrated $15-18$ that the electronic coherence makes the conductance deviate from the sinusoidal dependence on $\alpha$. We find that the electronic coherence in the multichannel regime can give rise to a nested peak structure. Our analysis indicates that the nested peak structure is due to the coherent Fabry-Perot-like interference in the multichannel regime. We remark that the nested peak structure was not found in previous studies, $7,8,19$ of a multichannel SFET since effects of the electronic coherence were not properly taken into account.

The paper is organized as follows. Section [I reports the numerical conductance calculation results of the conductance of multichannel SFETs as a function of the RSO coupling parameter $\alpha$. SFETs with various channel numbers are examined. When the spin injector and 
collector in SFETs are ideal, it is found that the conductance modulation ratio between the maximum and minimum conductances reduces as the number of channel increases. The decaying rate of the ratio is significantly larger for SFETs with larger $\alpha$. In order to gain insights into the numerical calculation results, energy dispersion relations and spin configurations of energy eigenstates are examined. Section [III focuses on the conductance properties of SFETs with rather small number of channels that exhibit a large conductance modulation ratio. It is found that the electronic coherence gives rise to a nested peak structure. Effects of an in-plane magnetic field are also addressed briefly and it is verified that the magnetic-field-induced peak splitting, reported previously for a single-channel SFET $\stackrel{18}{ }$, persists in the multichannel regime. Section[IV]addresses briefly behaviors of multichannel SFETs equipped with nonideal spin injectors and collectors, which consist of conventional metallic ferromagnet-thin insulator-2DEG hybrid structures. Scattering effects are also addressed briefly. Section V summarizes the paper.

\section{CONDUCTANCE OF MULTICHANNEL SFET}

Figure 1 shows a schematic drawing of a SFET with finite width $w$. Within the 2DEG in the $x y$-plane, the effective Hamiltonian of electrons reads

$$
H_{2 \mathrm{D}}=\frac{\mathbf{p}^{2}}{2 m^{*}}+\frac{\alpha}{\hbar}(\vec{\sigma} \times \mathbf{p}) \cdot \hat{\mathbf{z}}+V_{\mathrm{c}}(y),
$$

where $\mathbf{p}=\left(p_{x}, p_{y}\right)$ is the momentum operator within the 2DEG, $V_{\mathrm{c}}(y)$ is the transverse confinement potential with $V_{\mathrm{c}}(y)=0$ for $0<y<w$ and $V_{\mathrm{c}}(y)=\infty$ otherwise. Here $\vec{\sigma}=\left(\sigma_{x}, \sigma_{y}, \sigma_{z}\right)$ is the Pauli spin operator, and $\hat{\mathbf{z}}$ a unit vector perpendicular to the 2DEG.

In order to focus on the issue of the multichannel transport, we assume situations where effects of other practical problems are minimized; scattering by impurities and phonons is ignored in $H_{2}$ and ideal spin injection and detection are assumed. To be specific, an electron in the injector and collector is assumed to be subject to the

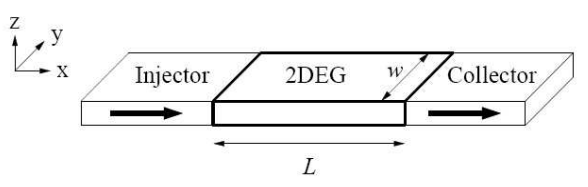

FIG. 1: Schematic diagram of a ballistic spin field effect transistor consisting of two-dimensional electron gas (2DEG) of finite width $w$ and two spin-selective electrodes (injector and collector), whose spins are assumed to be fully polarized along the $(+x)$-direction. The strength of the Rashba coupling can be tuned by external gates (not shown). following Hamiltonian,

$$
H_{\mathrm{inj} / \mathrm{col}}=\frac{\mathbf{p}^{2}}{2 m^{*}}+V_{\mathrm{c}}(y)-g \mu_{B} B_{\mathrm{ex}}\left(\sigma_{x}-1\right),
$$

where $B_{\text {ex }}$ is the effective exchange energy in the injector/collector, $g$ is the Lande's g-factor, and $\mu_{B}$ is the Bohr magneton. For sufficiently large $g \mu_{B} B_{\mathrm{ex}}(>0)$, electrons in the injector and collector are $100 \%$ spinpolarized along the $(+x)$-direction. Note that with this choice of $H_{\mathrm{inj} / \mathrm{col}}$, the Fermi wavelength of $(+x)$ polarized electron within the injector/collector matches perfectly with that within the 2DEG when $\alpha=0$ and thus the so-called conductance mismatch problem for the spin injection/detection does not appear for the 2DEGinjector(collector) interface. For nonzero $\alpha$, there is a weak mismatch in the Fermi wavelength but for the range of $\alpha$ values examined below $\left(\alpha<10^{-10} \mathrm{eVm}\right)$, the conductance mismatch problem turns out to be a weak effect (see Appendix $\mathrm{A}$ for details).

\section{A. Numerical conductance calculation}

For the numerical conductance calculation of the multichannel SFETs, we use the tight-binding (TB) Hamil-

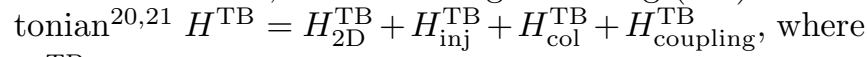
$H_{2 \mathrm{D}}^{\mathrm{TB}}$ amounts to the TB approximation of the Hamiltonian $H_{2 \mathrm{D}}$ [Eq. (1)] for the 2DEG,

$$
\begin{aligned}
H_{2 \mathrm{D}}^{\mathrm{TB}}= & 4 t \sum_{i=1}^{N_{x}} \sum_{j=1}^{N_{y}} \sum_{s_{z}= \pm 1} c_{i, j, s_{z}}^{\dagger} c_{i, j, s_{z}} \\
& -t \sum_{s_{z}= \pm 1}\left[\sum_{i=1}^{N_{x}-1} \sum_{j=1}^{N_{y}}\left(c_{i+1, j, s_{z}}^{\dagger} c_{i, j, s_{z}}+\text { H.c. }\right)\right. \\
& \left.+\sum_{i=1}^{N_{x}} \sum_{j=1}^{N_{y}-1}\left(c_{i, j+1, s_{z}}^{\dagger} c_{i, j, s_{z}}+\text { H.c. }\right)\right] \\
& +\lambda \sum_{s_{z}= \pm 1} \sum_{s_{z}^{\prime}= \pm 1}\left\{-\sum_{i=1}^{N_{x}-1} \sum_{j=1}^{N_{y}}\left[c_{i+1, j, s_{z}}^{\dagger}\left(i \sigma_{y}\right)^{s_{z}, s_{z}^{\prime}} c_{i, j, s_{z}^{\prime}}\right.\right. \\
& + \text { H.c. }] \\
& \left.+\sum_{i=1}^{N_{x}} \sum_{j=1}^{N_{y}-1}\left[-c_{i, j+1, s_{z}}^{\dagger}\left(i \sigma_{x}\right)^{s_{z}, s_{z}^{\prime}} c_{i, j, s_{z}^{\prime}}+\text { H.c. }\right]\right\}
\end{aligned}
$$

where $c_{i, j, s_{z}}$ is the annihilation operator of an electron at $\mathbf{r}_{i j}=a(i \hat{\mathbf{x}}+j \hat{\mathbf{y}})$ with spin $s_{z}$ along the $z$-axis, $a$ is the lattice spacing used for the TB approximation, and $N_{x}$ and $N_{y}$ are related to the length $L$ and width $w$ of the 2DEG via $a\left(N_{x}+1\right)=L$ and $a\left(N_{y}+1\right)=w$, respectively. With the choice $t=\hbar^{2} / 2 m^{*} a^{2}$ and $\lambda=\alpha / 2 a$, Eq. (3) reduces to Eq. (11) in the limit $a \rightarrow 0$. The TB Hamiltonians for the injector and collector are similarly 
given by

$$
\begin{aligned}
H_{\mathrm{inj}}^{\mathrm{TB}}= & 4 t \sum_{i=0}^{-\infty} \sum_{j=1}^{N_{y}} c_{i, j, s_{x}=1}^{\dagger} c_{i, j, s_{x}=1} \\
& -t\left[\sum_{i=-1}^{-\infty} \sum_{j=1}^{N_{y}}\left(c_{i+1, j, s_{x}=1}^{\dagger} c_{i, j, s_{x}=1}+\text { H.c. }\right)\right. \\
& \left.+\sum_{i=0}^{-\infty} \sum_{j=1}^{N_{y}-1}\left(c_{i, j+1, s_{x}=1}^{\dagger} c_{i, j, s_{x}=1}+\text { H.c. }\right)\right] \\
H_{\mathrm{col}}^{\mathrm{TB}}= & 4 t \sum_{i=N_{x}+1}^{\infty} \sum_{j=1}^{N_{y}} c_{i, j, s_{x}=1}^{\dagger} c_{i, j, s_{x}=1} \\
& -t\left[\sum_{i=N_{x}+1}^{\infty} \sum_{j=1}^{N_{y}}\left(c_{i+1, j, s_{x}=1}^{\dagger} c_{i, j, s_{x}=1}+\text { H.c. }\right)\right. \\
& \left.+\sum_{i=N_{x}+1}^{\infty} \sum_{j=1}^{N_{y}-1}\left(c_{i, j+1, s_{x}=1}^{\dagger} c_{i, j, s_{x}=1}+\text { H.c. }\right)\right],
\end{aligned}
$$

where $c_{i, j, s_{x}= \pm 1} \equiv\left(c_{i, j, s_{z}=1} \pm c_{i, j, s_{z}=-1}\right) / \sqrt{2}$. Note that the electron operators for $s_{x}=-1$ do not appear in $H_{\mathrm{inj} / \mathrm{col}}^{\mathrm{TB}}$, since the injector/collector is assumed to be $100 \%$ spin polarized along $(+x)$-direction. The coupling Hamiltonian between the 2DEG and the injector/collector is given by

$$
\begin{aligned}
H_{\text {coupling }}^{\mathrm{TB}}= & -t \sum_{j=1}^{N_{y}}\left(c_{1, j, s_{x}=1}^{\dagger} c_{0, j, s_{x}=1}\right. \\
& \left.+c_{N_{x}+1, j, s_{x}=1}^{\dagger} c_{N_{x}, j, s_{x}=1}+\text { H.c. }\right) .
\end{aligned}
$$

Note that electrons whose spin is pointing along the $(-x)$-direction are not allowed to hop between the 2DEG and the injector(collector). Note also that the hopping parameter $t$ in $H_{\mathrm{inj} / \mathrm{col}}^{\mathrm{TB}}$ is the same as that in $H_{2 \mathrm{D}}^{\mathrm{TB}}$. Thus when effects of $\alpha$ (or $\lambda$ ) on the Fermi wavelength is not significant, the conductance mismatch problem should be minimal (see Appendix $\mathrm{A}$ for detail), allowing ideal spin injection and detection.

The Landauer-Büttiker formalism is used for the numerical conductance calculation and the method in Ref. ${ }^{22}$ is used to evaluate matrix elements of related Greens' functions. The following parameters are used; $m^{*}=$ $0.04 \times m_{\text {electron }}$, where $m_{\text {electron }}$ is the free electron mass, and the Fermi energy $E_{F}=0.103 \mathrm{eV}$, which, in the absence of $\alpha$ and $V_{\mathrm{c}}$, amounts to the Fermi wavelength $2 \pi / k_{F}=191 \mathrm{~A}$ and the electron density of $n_{s}=$ $1.72 \times 10^{12} \mathrm{~cm}^{-2}$ in the 2DEG. For the TB approximation, we choose $a=\left(2 \pi / k_{F}\right) / 9.95$. Thus the hopping energy $t=\hbar^{2} / 2 m^{*} a^{2}$ becomes $0.259 \mathrm{eV}$.

Below the conductance is first evaluated in two windows of $\alpha, \quad(0 \sim 10) \times 10^{-12} \mathrm{eVm}$ and $(45 \sim 55) \times 10^{-12}$
$\mathrm{eVm}$. In various candidate systems for the SFET such as $\operatorname{InGaAs} / \operatorname{InAlAs} s^{2}$, InAs/GaSb $\stackrel{11}{ }$, InGaAs/InP $\stackrel{12}{ }$, and InAs/AlSb $\frac{13}{2}, \alpha$ typically falls within the first window. A recent calculation 23 also reported that for III-V quantum wires, $\alpha$ is typically in this weak coupling regime. Search for systems with higher $\alpha$ is in progress and effects of strong RSO coupling on the energy spectrum are addressed theoretically 24 . For $\mathrm{CdTe} / \mathrm{HgTe} / \mathrm{CdTe}$, for instance, values up to $\sim 45 \times 10^{-12} \mathrm{eVm}$ has been reported $\stackrel{14}{ }$, which motivates the second window. For a SFET with relatively short $L$, the conductance is evaluated in a much wider window of $\alpha,(0 \sim 100) \times 10^{-12} \mathrm{eVm}$.

Figure 2 shows the evolution of the conductance $G$ as a function of $\alpha$ for the SFET with 3, 5, 10, and 20 channels, respectively. For the range of $\alpha$ examined below, the number of channels $N_{\mathrm{ch}}$ is related to the width $w$ of the
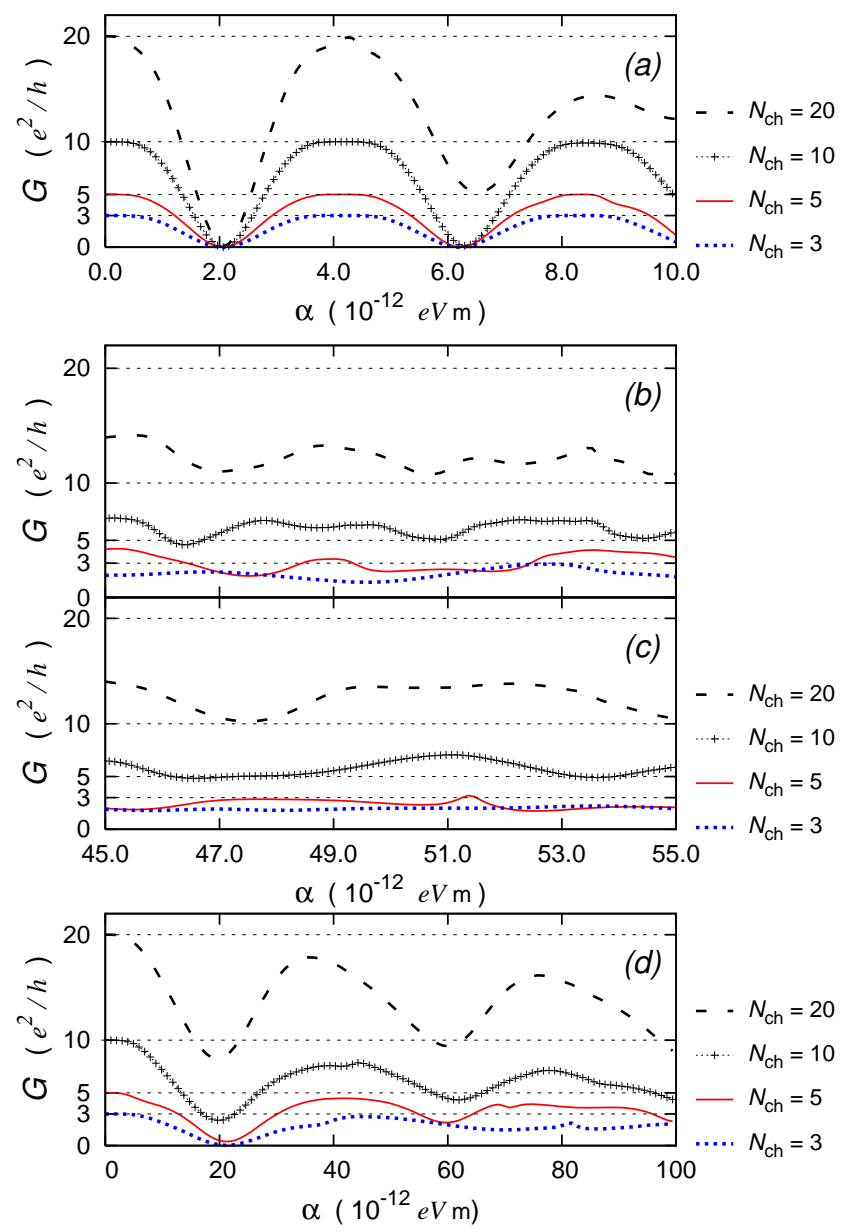

FIG. 2: (Color online) Conductance $G$ of a ballistic multichannel SFET as a function of the RSO coupling parameter $\alpha$ at the temperature $T=0$. Ideal spin injection and detection are assumed. The following parameters are used; the effective mass $m^{*}=0.04 \times m_{\text {electron }}$, where $m_{\text {electron }}$ is the free electron mass, and the electron density $n_{s}=1.72 \times 10^{12} \mathrm{~cm}^{-2}$. The length $L$ of the 2DEG is $1.44 \mu \mathrm{m}$ for (a) and (b), $0.8 \mu \mathrm{m}$ $(\approx 0.556 \times 1.44 \mu \mathrm{m})$ for $(\mathrm{c})$, and $0.1 \times 1.44 \mu \mathrm{m}$ for $(\mathrm{d})$. 
2 DEG via the relation ${ }^{20} N_{\mathrm{ch}}=\operatorname{Int}\left(k_{F} w / \pi\right)$, where $\operatorname{Int}(x)$ denotes the largest integer not exceeding $x$. The length $L$ of the 2DEG is assumed to be $1.44 \mu \mathrm{m}$ for (a) and (b), and $0.8 \mu \mathrm{m}(\approx 0.556 \times 1.44 \mu \mathrm{m})$ for $(\mathrm{c})$, and $0.1 \times$ $1.44 \mu \mathrm{m}$ for (d), respectively. In Fig. 2(a), the SFET is operated in the $\alpha$ range from 0 to $10 \times 10^{-12} \mathrm{eVm}$. To estimate the spin precession angle, we use the formula $2 m^{*} \alpha L / \hbar^{2}$. Though this formula is derived in the singlechannel limit, it may still be useful to estimate the spin precession in multichannel SFETs. Then the average spin precession angle, estimated from the formula $2 m^{*} \alpha L / \hbar^{2}$ with the average $\alpha=5.0 \times 10^{-12} \mathrm{eVm}$, is around $1.2 \times 2 \pi$, and the variation of the spin precession angle within the specified range is $\pm 1.2 \times 2 \pi$. Note that the SFET exhibits an almost ideal conductance modulation in the sense that the maximum of $G$ reaches almost $\left(e^{2} / h\right) N_{\mathrm{ch}}$ and the minimum of $G$ reaches almost zero. The conductance modulation behavior becomes, however, less ideal with the increase of $N_{\mathrm{ch}}$. For $N_{\mathrm{ch}}=20$, for instance, Fig. 22(a) already shows some deviation from the ideal behavior. Figure 3(a) shows, as a function of $N_{\mathrm{ch}}$, the ratio between $G_{\max }$ and $G_{\min }$, where $G_{\max }$ and $G_{\min }$ are the maximum and minimum values of $G$ in the interval $1.8 \times 10^{-12} \mathrm{eVm}$ $<\alpha<4.5 \times 10^{-12} \mathrm{eVm}$ in Fig. 2(a). It clearly shows the decay of the ratio with $N_{\mathrm{ch}}$.

In Fig. 2(b) (again with $L=1.44 \mu \mathrm{m}$ ), the SFET is operated in the $\alpha$ range $(45 \sim 55) \times 10^{-12} \mathrm{eVm}$. Note that the width of the $\alpha$ range is the same as that in Fig. 2(a). Since $\alpha$ is larger than the values in Fig. 2(a), the spin precession angle will be larger. The average spin precession angle in this range, estimated from the formula $2 m^{*} \alpha L / \hbar^{2}$ with the average $\alpha=50 \times 10^{-12} \mathrm{eVm}$, is around $12 \times 2 \pi$, about 10 times larger than in Fig. 2(a). On the other hand, the variation of the spin precession angle within the specified range is about $\pm 1.2 \times 2 \pi$, same as in Fig. 2(a). Note that the conductance modulation behavior is now much less ideal; the maximum and minimum of $G$ deviate considerably from $\left(e^{2} / h\right) N_{\text {ch }}$ and 0 , respectively. Moreover the conductance oscillation with $\alpha$ becomes somewhat irregular. Figures 3 (b) shows, as a function of $N_{\mathrm{ch}}$, the ratio between $G_{\max }$ and $G_{\min }$ for the SFET in Fig. 2(b). Here $G_{\max }$ and $G_{\min }$ are evaluated in the $\alpha$ range $(48.5 \sim 51.5) \times 10^{-12} \mathrm{eVm}$. Note that the decay of the ratio with increasing $N_{\mathrm{ch}}$ is much faster than that in Fig. 3(a). For small $N_{\text {ch }}$ around 5, the ratio $G_{\max } / G_{\min }$ already drops to of order unity.

One intuitive explanation for the much less ideal behavior in Figs. 2(b) and 3(b), compared to Figs. 2(a) and 3(a), is fluctuations of the spin precession rates from channel to channel. For example, if the spin precession angles per length deviate from $2 m^{*} \alpha / \hbar^{2}$ and the deviations fluctuate from channel to channel, the conductance modulation may become less ideal. To test this possibility, we examine a SFET with shorter $L$ since effects of the channel-to-channel fluctuation are expected to be weakened with decreasing $L$. In Fig. 2(c), the length $L$ of the $2 \mathrm{DEG}$ is reduced to $0.8 \mu \mathrm{m}(\approx 0.556 \times 1.44 \mu \mathrm{m})$. When the SFET is operated in the same $\alpha$ range as in Fig. 2(b),

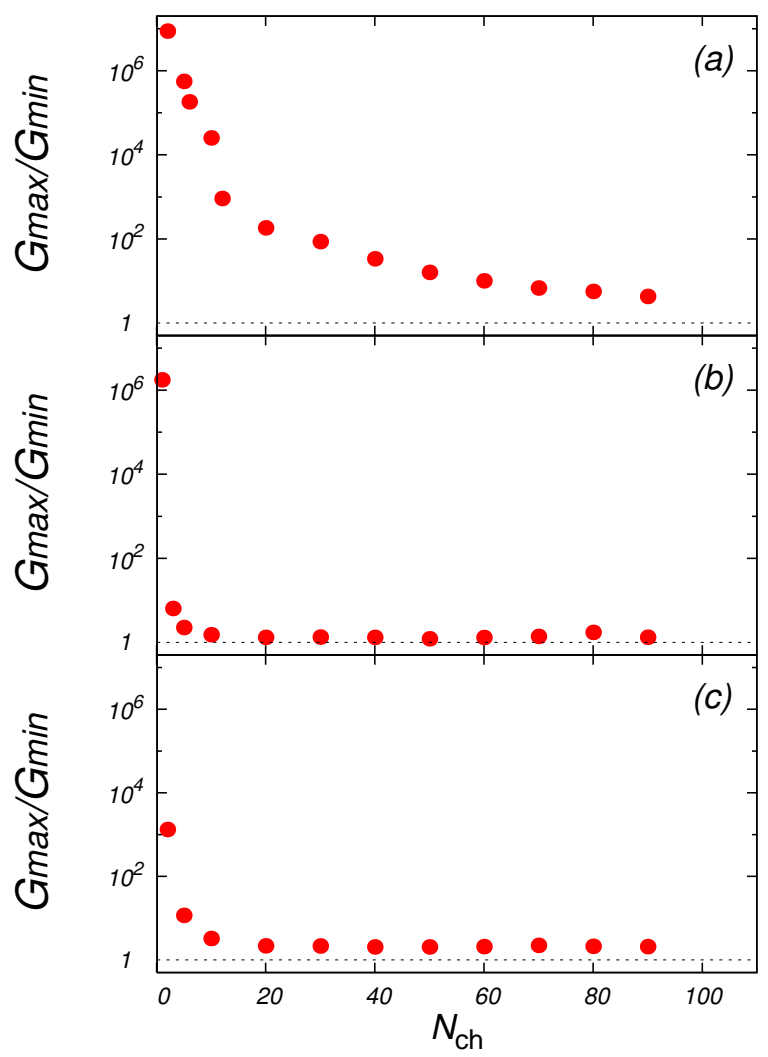

FIG. 3: (Color online) The ratio between the maximum conductance $G_{\max }$ and the minimum conductance $G_{\min }$ as a function of the number of channels $N_{\text {ch }}$. The panels (a), (b), and (c) correspond to the situations in Fig. 2(a), (b), and (d), respectively. The parameters are the same as those for Fig. 2

the average spin precession is estimated to $6.7 \times 2 \pi$, which is about $44 \%$ smaller than that in Fig. 2(b), and the variation of the spin precession angle within the specified range is estimated to $\pm 0.7 \times 2 \pi$. Figure 2(c) shows that the conductance modulation is still far from ideal despite the $44 \%$ reduction in the average spin precession angle.

In Fig. 2(d), the length $L$ of the 2DEG is further reduced to $0.1 \times 1.44 \mu \mathrm{m}$, one tenth of that in Fig. 2 (a). When the SFET is operated in the range $0<\alpha<100 \times$ $10^{-12} \mathrm{eVm}$, which is 10 times larger than in Fig. 2(a), the average spin precession angle and the variation of the angle within the specified $\alpha$ range are exactly same as those in Fig. 2(a). Thus the situations in Figs. 2(a) and (d) are identical as far as the expression $2 m^{*} \alpha L / \hbar^{2}$ for the spin precession angle is concerned. The numerical calculation result in Fig. 2(d) indicates however that while the conductance modulation behavior is somewhat improved compared to those in Figs. 2(b) and (c), it is still less ideal than that in Fig. 2(a). Figure 3(c) shows the $N_{\mathrm{ch}}$-dependence of the ratio $G_{\max } / G_{\min }$ for the situation in Fig. 2(d), where $G_{\max }$ and $G_{\min }$ are evaluated in the interval $18 \times 10^{-12} \mathrm{eV}<\alpha<45 \times 10^{-12} \mathrm{eVm}$. 
Note that the decay of the ratio is still much faster than in Fig. 3(a). This result leads one to conclude that the channel-to-channel fluctuations of the spin precession angles are not the main reason for the deviations from the ideal behavior.

\section{B. Transport channels}

In order to gain an insight into the numerical results in Figs. 2 and 3, it is useful to examine energy eigenfunctions and eigenenergies of $H_{2 \mathrm{D}}$ [Eq. (1)]. Let $\Psi(x, y)=e^{i k_{x} x} \psi(y)$ denote a eigen wavefunction with energy $E$. Due to the translational symmetry along the $x$-direction within the 2DEG, it can be expressed as a superposition of four plane waves $\Psi=\sum_{j=1}^{4} c_{j} \Psi_{\mathbf{k}_{j}}$, where the plane wave $\Psi_{\mathbf{k}_{j}}(x, y)=e^{i \mathbf{k}_{j} \cdot \mathbf{r}} u_{j}\left(\mathbf{k}_{j}\right)$ is an eigenstate of $H_{2 \mathrm{D}}$ in the absence of $V_{\mathrm{c}}(y)$. Here all four wavevectors $\mathbf{k}_{j}=\left(k_{x, j}, k_{y, j}\right)$ share the same longitudinal component $k_{x, j}=k_{x}$ and their transverse components $k_{y, j}$ are determined from the relation $\hbar^{2}\left(k_{x, j}^{2}+\right.$ $\left.k_{x, j}^{2}\right) / 2 m^{*}+(-1)^{j} \alpha\left(k_{x, j}^{2}+k_{y, j}^{2}\right)^{1 / 2}=E$, which is the energy-dispersion relation in the absence of $V_{\mathrm{c}}(y)$ (see Fig. (4). The spinor $u_{j}(\mathbf{k})$ is given by

$$
u_{j}(\mathbf{k})=\frac{1}{\sqrt{2}}\left(\begin{array}{c}
1 \\
(-1)^{j+1} i e^{i \varphi(\mathbf{k})}
\end{array}\right)
$$

where $\varphi(\mathbf{k})$ is defined by $\cos \varphi(\mathbf{k})=k_{x} /\left(k_{x}^{2}+k_{y}^{2}\right)^{1 / 2}$ and $\sin \varphi(\mathbf{k})=k_{y} /\left(k_{x}^{2}+k_{y}^{2}\right)^{1 / 2}$. Then the four constraints from the boundary conditions $\Psi(x, 0)=\Psi(x, w)=0$ fix the four coefficients $c_{j}$, and the exact energy-dispersion relations and the eigen wavefunctions can be obtained. This procedure can be simplified further by using additional symmetries of $H_{2 \mathrm{D}}$. See Appendix $\mathrm{B}$ for further details on the symmetries, and Appendix $\mathrm{C}$ for the use of the symmetries for the evaluation of the exact energydispersion relation and the eigen wavefunctions.

The red dotted lines in Fig. [5]denote the exact energydispersion relation for various situations. In comparison, the perturbatively obtained energy-dispersion relation (up to the second order in $\alpha$ ),

$$
E_{n, i}=\frac{\hbar^{2} k_{x}^{2}}{2 m^{*}}+\mathcal{E}_{n}+(-1)^{i} \alpha k_{x}-\frac{m^{*} \alpha^{2}}{2 \hbar^{2}},
$$

is also depicted (blue solid lines) in Fig. 5 Here $\mathcal{E}_{n} \equiv$ $\hbar^{2} k_{n, y}^{2} / 2 m^{*}$ is the energy of the $n$-th $(n=0,1,2, \cdots)$ excitation mode in the transverse direction, $k_{n, y} \equiv(n+1) \pi / w$, and $i=1$ or 2. Equation (8) is obtained by treating the RSO coupling term in $H_{2 \mathrm{D}}$ as a perturbation. Further details on the calculation of the energy dispersion relation via the second-order degenerate perturbation theory are given in Appendix D.

Figure 6 shows the local spin directions of the selected eigen wavefunctions at the Fermi level $E_{F}$. The width $w$ and the RSO parameter $\alpha$ for Figs. 6(a), (b), (c), and (d) are the same as those for Figs. 5(a), (b), (c), and (d), respectively. The local spin direction of an energy eigen wavefunction is independent of $x$ due to the translational symmetry of $H_{2 \mathrm{D}}$ along the $x$-direction. Also it can be shown that it is always perpendicular to the $x$-axis and remains within the $y z$-plane since $H_{2 \mathrm{D}}$ remains invariant under the symmetry operator $\Theta \Pi_{x} \mathcal{D}_{\text {spin }}(\hat{\mathbf{x}}, \pi)$ (see Appendix B for further details), where $\Theta$ is the timereversal operator, $\Pi_{x}$ is the mirror reflection operator with respect to the $y z$-plane in the orbital space, and $\mathcal{D}_{\text {spin }}(\hat{\mathbf{x}}, \pi)$ is the rotation operator with respect to the $x$-axis by the angle $\pi$ in the spin space. In each upper panel in Figs. 6(a), (b), (c), and (d), the arrows indicate the local spin direction as a function of $y$ for six selected exact eigen wavefunctions. The length of the arrows is proportional to $\sqrt{\Psi^{\dagger}(x, y) \Psi(x, y)}$, which is independent of $x$. The six states in Figs. 6(a) and (c) are the six eigenstates in Figs. [5(a) and (c) at $E_{F}=0.398 t=0.103$ $\mathrm{eV}$ with increasing order of $k_{x}>0$. On the other hand, the six states in Figs. 6(b) and (d) are the eigenstates in Figs. 5 (b) and (d) at $E_{F}$ with the smallest, 2nd smallest, 7th smallest, 8th smallest, 13th smallest, and 14 th smallest $k_{x}$ 's (ordered according to the exact $k_{x}$ values). The lower panels in Figs. 6(a), (b), (c), and (d) show the local spin angle $\theta$ as a function of $y$, where $\theta$ is $-\pi / 2,0, \pi / 2, \pm \pi$ when the local spin direction is pointing $-y,+z,+y,-z$ axis, respectively. Here $\theta$ is related to the local spin direction via the spinor representation $\left(\cos \frac{\theta}{2} \chi_{\uparrow}+i \sin \frac{\theta}{2} \chi_{\downarrow}\right)$ of a local spin direction that lies within the $y z$-plane, and $\chi_{\uparrow}=\left(\begin{array}{l}1 \\ 0\end{array}\right), \chi_{\downarrow}=\left(\begin{array}{l}0 \\ 1\end{array}\right)$ are the spinors pointing along the $( \pm z)$-directions. In each lower panel of Figs. 6(a), (b), (c), and (d), there are three subpanels, each of which shows the profiles of

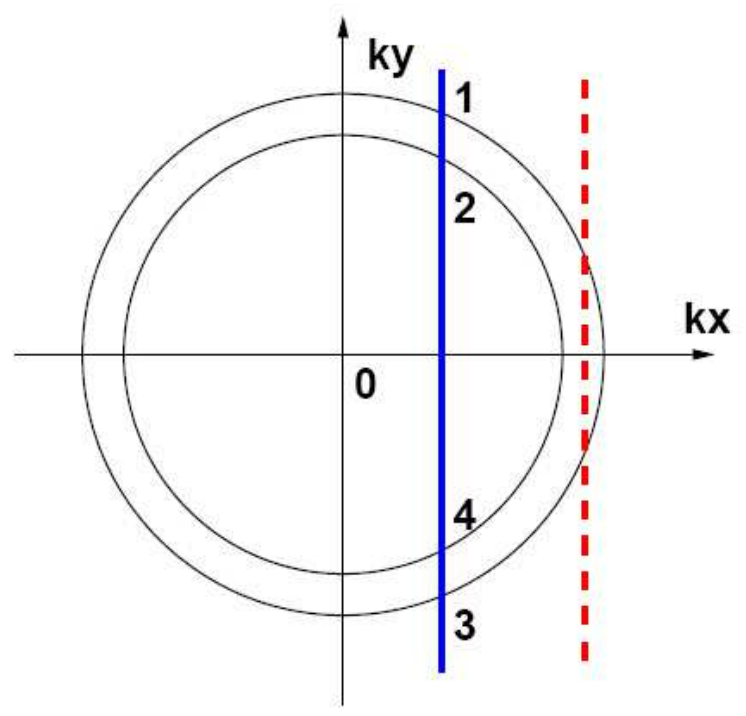

FIG. 4: (Color online) Four solutions $k_{y, j}(j=1,2,3,4)$ of $\hbar^{2}\left(k_{x}^{2}+k_{y, j}^{2}\right) / 2 m^{*}+(-1)^{j} \alpha\left(k_{x}^{2}+k_{y, j}^{2}\right)^{1 / 2}=E$, which is the energy dispersion relation in the absence of $V_{\mathrm{c}}(y)$. Note that for certain $k_{x}$ (denoted by the red dashed line), two solutions become purely imaginary numbers. 
$\theta(y)$ 's for two selected energy eigen wavefunctions; $\theta$ 's in the left/middle/right subpanel correspond to the two leftmost/central/rightmost spin textures in the upper panel. In the lower panels, blue dotted lines represent the exact results (see Appendix C for further details) and red solid lines are from the perturbatively obtained eigen wavefunctions calculated up to the second order in $\alpha$ (see Appendix $\mathrm{D}$ for further details).

In Figs. 5 and 6. two representative values of $\alpha$, $8.3 \times 10^{-12} \mathrm{eVm}\left[(\mathrm{a})\right.$ and (b)] and $49.8 \times 10^{-12} \mathrm{eVm}$ $[(c)$ and (d)], are used. Figures 5 (a) and 6 (a) show the result for the smaller $\alpha$ value and $N_{\mathrm{ch}}=3$. Note that the perturbative result for the energy dispersion relation is in excellent agreement with the exact result. Equation (8) from the perturbation theory indicates that the energy dispersion relations for all channel $n$ are identical to each other up to the parallel shift by $\mathcal{E}_{n}$ and remain parabolic. Moveover Fig. 6(a) indicates that the spin direction is essentially equal to the $(+y)$ or $(-y)$-direction, same as in

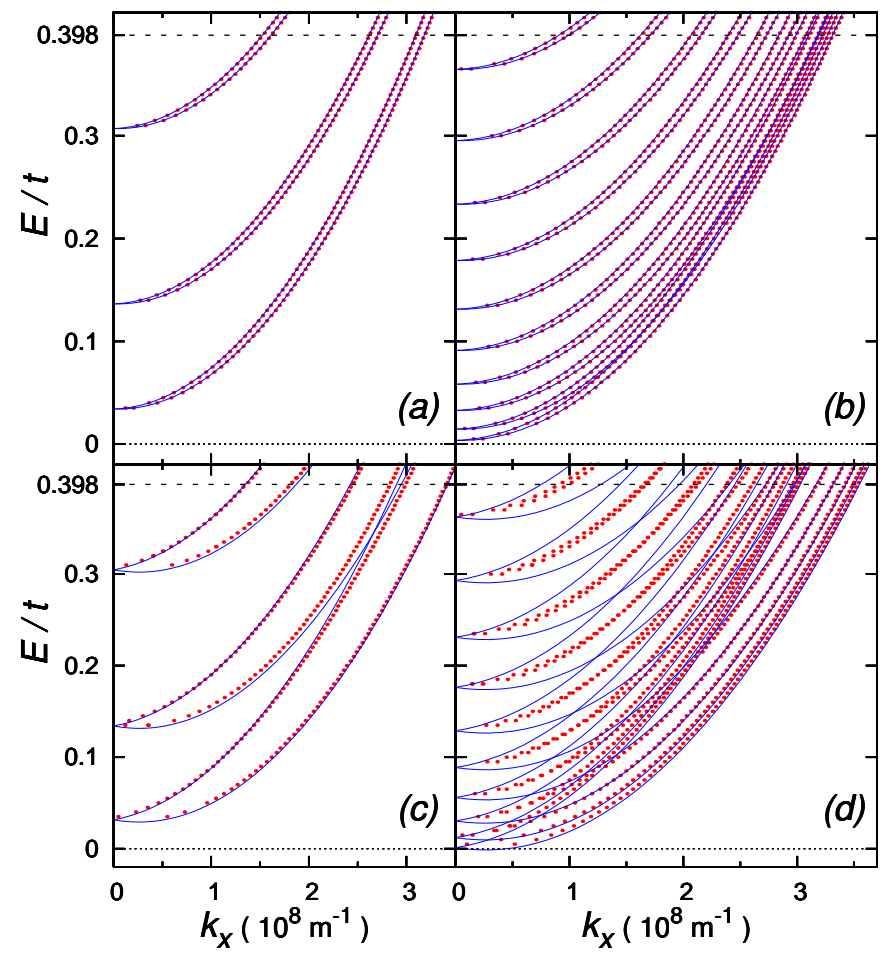

FIG. 5: (Color online) The energy dispersion relations in a Rashba spin-orbit (RSO) coupled system with hard wall confinement. Red dotted lines represent the exact dispersion relations, while blue solid lines the perturbative results, Eq. (8). The two types of the lines overlap almost perfectly in (a) and (b). The RSO coupling parameter $\alpha$ and the number of channels $N_{\text {ch }}$ are $\alpha=8.3 \times 10^{-12} \mathrm{eVm}, N_{\mathrm{ch}}=3$ in (a), $\alpha=8.3 \times 10^{-12} \mathrm{eVm}, N_{\mathrm{ch}}=10$ in (b), $\alpha=49.8 \times 10^{-12}$ $\mathrm{eVm}, N_{\mathrm{ch}}=3$ in (c), and $\alpha=49.8 \times 10^{-12} \mathrm{eVm}, N_{\mathrm{ch}}=10$ in (d), respectively. The width $w$ is $32.7 \mathrm{~nm}$ for (a) and (c), and $100.0 \mathrm{~nm}$ for (b) and (d). The energy is given in units of $t=0.259 \mathrm{eV}$. The horizontal dashed lines denote the Fermi energy $E_{F}=0.398 t=0.103 \mathrm{eV}$. the ideal single-channel SFET. Thus the SFET for the situation in Fig. 5(a) and 6(a) should show an almost ideal behavior, which is indeed the case as demonstrated in Figs. 2(a) for $N_{\mathrm{ch}}=3$. The channel-to-channel fluctuations of the spin precession angle should be negligible.

Figures 5(b) and 6(b) show the result for $\alpha=8.3 \times$ $10^{-12} \mathrm{eVm}$ and $N_{\mathrm{ch}}=10$. The exact energy dispersion relations are again excellently fitted by the perturbative results [Eq. [8] ], which are purely parabolic. The local spin directions, on the other hand, show noticeable deviations from the ideal $( \pm y)$-directions. The lower subpanels in Fig. 6(b) show that near the "nodal" points, where the length of the spin arrows in the upper panel of Fig. 6(b) almost vanishes, $\theta(y)$ changes rapidly by $2 \pi$, forming "phase-slip"-like structures. The phase-slip-like structure near the nodal points is more clearly visible in
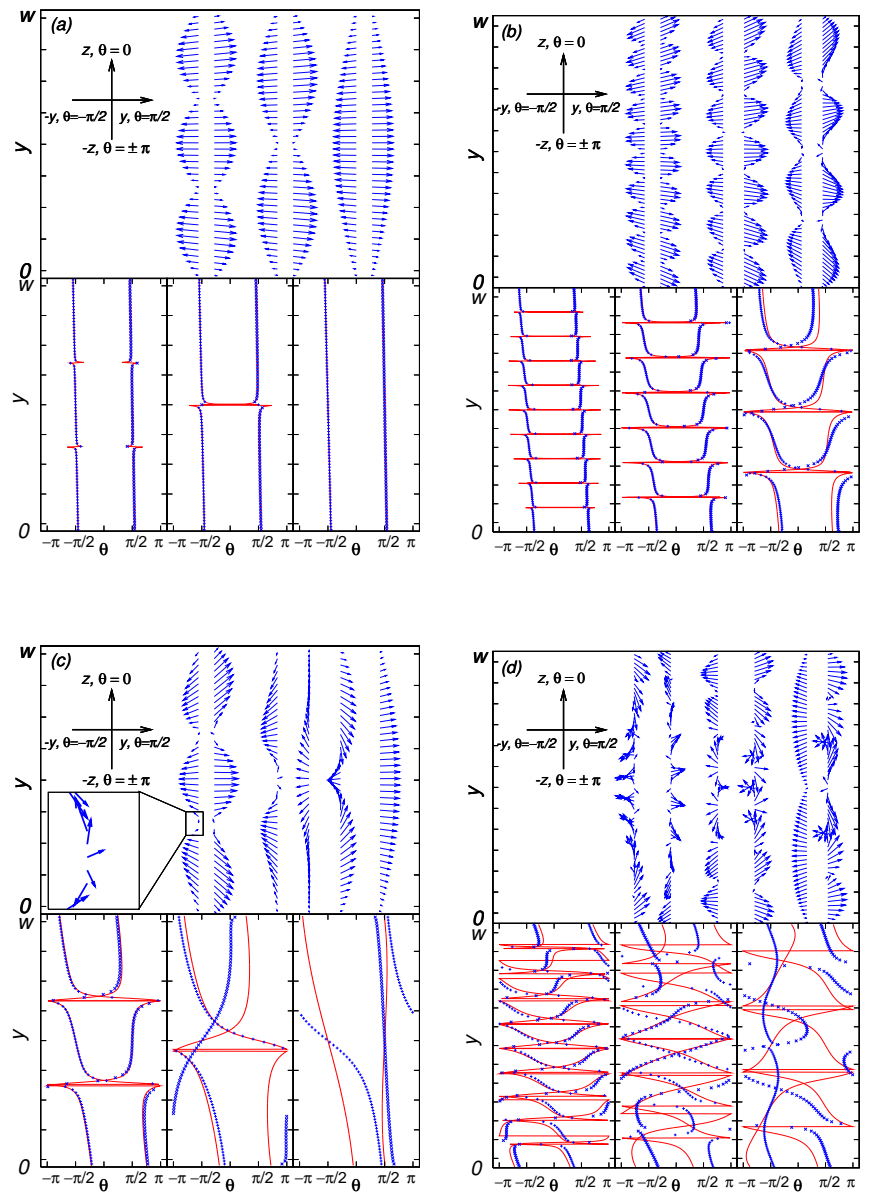

FIG. 6: (Color online) Spin texture along the transverse direction for selected states at $E_{F}$. The parameters $m^{*}, \alpha, w$ and $N_{\text {ch }}$ in (a), (b), (c) and (d) are the same as those in Figs. [5(a), (b), (c) and (d), respectively. In the lower panels, the blue dotted lines represent the exact results while the red solid lines the perturbative results. Inset in the upper panel of (c) shows the magnified structure near the points where $\sqrt{\Psi^{\dagger}(x, y) \Psi(x, y)}$ almost vanishes. 
the magnified plot in the inset of Fig.6(c). Superimposed over the phase-slip-like structures, there is also a gradual drift of $\theta(y)$ with the increase of $y$. To understand the origin of the deviations, the eigen wavefunctions are perturbatively evaluated (see Appendix (D). Up to the first order in $\alpha$, the perturbative calculation for the reduced wavefunction $\psi(y)\left[\Psi(x, y)=e^{i k_{x} x} \psi(y)\right]$ results in

$$
\psi_{n, i}(y)=\psi_{n, i}^{(0)}(y)+(-1)^{i+1} \frac{m^{*} \alpha(y-w / 2)}{\hbar^{2}} \psi_{n, i}^{(0)}(y)
$$

where $n$ and $i$ are defined in the same way as in Eq. (8), and $\bar{i}=2$ for $i=1$ and $\bar{i}=1$ for $i=2$. Here the zeroth order reduced wavefunctions are $\psi_{n, 1}^{(0)}(y)=\phi_{n}(y) \cdot\left(\chi_{\uparrow}+\right.$ $\left.i \chi_{\downarrow}\right) / \sqrt{2}$ and $\psi_{n, 2}^{(0)}(y)=\phi_{n}(y) \cdot\left(\chi_{\uparrow}-i \chi_{\downarrow}\right) / \sqrt{2}$ respectively, where $\phi_{n}(y) \equiv \sqrt{2 / w} \sin k_{n, y} y$. From these expressions, one obtains simple expressions for $\theta$,

$$
\begin{aligned}
& \theta_{n, i=1}(y)=\frac{\pi}{2}-\frac{2 m^{*} \alpha}{\hbar^{2}}(y-w / 2)+\mathcal{O}\left(\alpha^{2}\right), \\
& \theta_{n, i=2}(y)=-\frac{\pi}{2}-\frac{2 m^{*} \alpha}{\hbar^{2}}(y-w / 2)+\mathcal{O}\left(\alpha^{2}\right),
\end{aligned}
$$

which has been reported previously 25 . Note that while the zeroth order terms in $\alpha$ are $y$-independent constants, $\pm \pi / 2$, corresponding to the ideal $( \pm y)$-directions, the first order terms in $\alpha$ are proportional to $y-w / 2$, explaining the gradual drift of $\theta$ with $y$. In order to gain an insight into the phase-slip-like structure, we next examine the second order corrections to $\psi(y)$. Since the first order correction in Eq. (9) identically vanishes at the nodal points $\left(\sin k_{n, y} y=0\right)$ of $\psi_{n, i}^{(0)}$, the second order corrections are the first nonvanishing terms near those nodal points. Moreover since $\psi=0$ is a topologically singular point for the local spin angle $\theta$, even a small and smooth corrections to $\psi$ can result in a rapid change of $\theta$ by $2 \pi$. The local spin angles evaluated from the second order perturbation theory are shown (red solid lines) in the lower subpanels in Fig. 6(b), indeed reproducing the phase-slip-like structure. The perturbative results are in reasonable agreement with the exact results. The expressions for the second order corrections to $\psi$ are rather lengthy [Eqs. (D14) and (D15)] and given in the Appendix D only. We remark that the gradual drift and the phase-slip-like structures are also present in Fig. 6(a), though they are much weaker. Since they can be explained within the perturbation theory, the strength of their effects is expected to depend on the magnitude of the small expansion parameter of the perturbation theory, which is $2 m^{*} \alpha w / \hbar^{2}$ according to Eqs. (9), (D14), and (D15). Then recalling that the product $2 m^{*} \alpha w / \hbar^{2}$ is about 0.28 for the 3 channel system and about 0.86 for the 10 channel system, the difference between Figs. 6)(a) and (b) can be explained. This analysis also implies that the deviations of the local spin configuration from the ideal spin directions will be magnified with the increase of $N_{\mathrm{ch}}(\propto w)$. Since these deviation will certainly cause the conductance to deviate from the ideal SFET behavior based on the ideal spin directions, this analysis also provides an explanation for the initial decay of the conductance modulation ratio with the increase of $N_{\text {ch }}$ demonstrated in Figs. 2(a) and 3(a).

Figures 5(c) and 6)(c) show the result for the larger $\alpha=49.8 \times 10^{-12} \mathrm{eVm}$ and $N_{\mathrm{ch}}=3$ with the product $2 m^{*} \alpha w / \hbar^{2} \approx 1.7$, which is about two times larger than in Figs. 5)(b) and 6(b). Figure 5(c) shows that now some energy dispersion relations noticeably deviate from the parabolic behaviors. While many subbands are still well fitted by the parabolic perturbative results, the two subbands, which are predicted by the perturbation calculation to cross near $k_{x} \approx 2.5 \times 10^{8} \mathrm{~m}^{-1}$, are affected by the subband mixing and the avoided crossing structure appears, which goes beyond the perturbation calculation in Appendix D. At $E_{F}$, the states with the second and third largest $k_{x}$ 's are affected by the avoided crossing and their dispersions deviate from the parabolic dependence, modifying the spin precession rates of the involved channels as demonstrated recently 26 . The avoided crossing also affects the local spin angle. For those two states affected by the avoided crossing, the exact local spin angle profiles deviate considerably from the perturbative results [lower subpanels of Fig. 66(c)]. For the other four states, the exact local spin profiles are reasonably well fitted by the perturbative results. Note that the gradual drift of $\theta$ and the phase-slip-like structure are more evident than in Fig. 6(b), which is natural in view of the about two-fold increase of the product $2 m^{*} \alpha w / \hbar^{2}$. Based on this analysis, we attribute the reduced conductance modulation ratio in Figs. 2(b) and 3(b) (even for small $N_{\text {ch }}$ such as 3) jointly to the perturbative spin configuration change and the avoided crossing. We remark that the avoided crossing in fact occurs in Fig. 5)(b) as well, though not clearly visible in the figure since its effects on the energy dispersions are restricted to rather narrow ranges of $k_{x}$. Furthermore the avoided crossing appears far below $E_{F}$, so that its effects on the conductance is negligible.

Figures 5(d) and 6)(d) show the result for $\alpha=49.8 \times$ $10^{-12} \mathrm{eVm}$ and $N_{\mathrm{ch}}=10$ with $2 m^{*} \alpha w / \hbar^{2} \approx 5.2$. It turns out that most subbands are affected by the avoided crossing and the agreement between the perturbative and exact dispersion relations is poor. The agreement between the perturbative and exact spin configuration is again poor for many states at $E_{F}$. The abundance of the avoided crossing explains the strongly suppressed conductance modulation ratio of the SFET operated with larger $\alpha$ [Figs. 3(b) and (c)].

\section{COHERENT SFET}

The electronic coherence may give rise to interesting effects in mesoscopic systems ${ }^{20,27}$. For a single channel system, it has been predicted ${ }^{15-18}$ that due to the electron coherence, the conductance behavior of a SFET deviates from the conventional sinusoidal dependence $\cos ^{2}\left(m^{*} \alpha L / \hbar^{2}\right)$. In this section, we investigate effects 

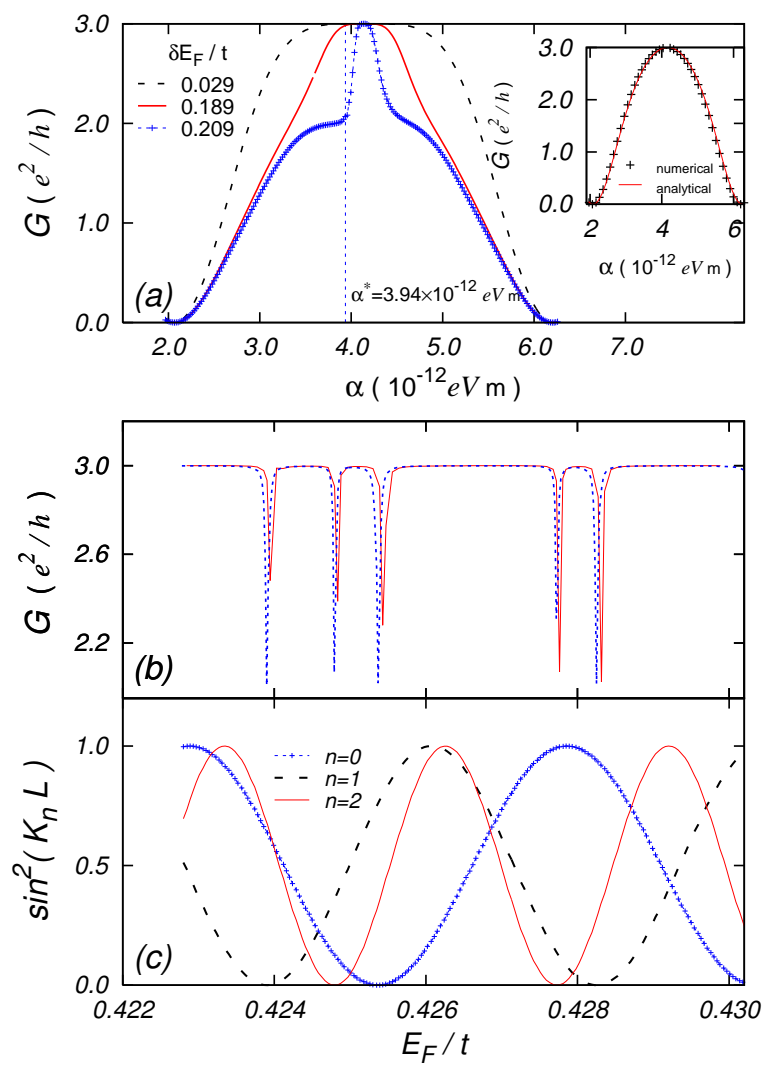

FIG. 7: (Color online) (a) Variations of the conductance profile with $E_{F}$ for a SFET with $N_{\text {ch }}=3$ and $L=1.44 \mu \mathrm{m}$ at the temperature $T=0 . \delta E_{F}$ 's are measured with respect to the reference energy $0.398 t=0.103 \mathrm{eV}$. Inset : The finite temperature conductance $G(\alpha)$ at $k_{B} T=10 \hbar v_{F} / L$ and $\delta E_{F} / t=$ 0.209 where $v_{F} \equiv \hbar\left(K_{0}+K_{1}+K_{2}\right) / 3 m^{*}$. Note that the numerical result is in excellent agreement with the analytic high temperature expression ${ }^{28}$. (b) The red solid line represents the numerically calculated $G\left(\alpha^{*}=3.94 \times 10^{-12} \mathrm{eVm}\right)$ as a function of $E_{F}$ at $T=0$. Here $\alpha^{*}$ [vertical dotted line in (a)] is close to the conductance maximum point. Thus dips in the $G\left(\alpha^{*}\right)$ graph imply the appearance of the nested peak structure at the corresponding $E_{F}$. For comparison, the prediction of the formula [Eq. (12)] is also shown (blue dashed line). (c) $\sin ^{2}\left(K_{n} L\right)$ for $n=0,1$, and 2. The comparison between (b) and (c) indicates that the dip positions of $G\left(\alpha^{*}\right)$ coincide with the points where one of $\sin ^{2}\left(K_{n} L\right)$ 's becomes zero.

of the coherence on multichannel SFETs. In particular, we focus on situations with $N_{\text {ch }} \lesssim 10$ and $\alpha \lesssim 10 \times 10^{-12}$ eVm since for larger $N_{\mathrm{ch}}$ and $\alpha$, the coherence effects will be mixed with other effects such as nonideal spin configuration and avoided crossing, making clear identification of the coherence effects difficult.

Hints of the coherence effects appear already at Fig. 2(a). A close look reveals the deviation of the line profile $G(\alpha)$ from the sinusoidal behavior $\propto$ $\cos ^{2}\left(m^{*} \alpha L / \hbar^{2}\right)$. The evolution of $G(\alpha)$ with the variation of the Fermi energy $E_{F}$ illustrates the deviation more clearly. Figure $7($ a) shows $G(\alpha)$ of a SFET with $N_{\mathrm{ch}}=3$ for a number of $E_{F}$ 's. $\delta E_{F}$ 's in Fig. 7(a) are measured with respect to the reference energy 0.103 $\mathrm{eV}=0.398 t$. While $G(\alpha)$ is not sensitive to $E_{F}$ near the conductance maximum (near $\alpha=4.2 \times 10^{-12} \mathrm{eVm}$ ) and the minimum (near $\alpha=2.2 \times 10^{-12} \mathrm{eVm}$ and $\left.6.2 \times 10^{-12} \mathrm{eVm}\right)$, the profiles of $G(\alpha)$ including the full-width-at-half-maximum (FWHM) exhibit considerable dependence on $E_{F}$. Moreover near certain special $E_{F}$ 's such as $\delta E_{F} / t=0.209, G(\alpha)$ shows a nested peak structure, where a small peak appears on top of a bigger background peak. These results are in clear contrast to the result in Ref $\frac{19}{2}$, which reports the absence of the $E_{F}$-dependence when $2 m^{*} \alpha w / \hbar^{2} \ll 2 \pi$. While our conductance calculation takes into account all three parts (2DEG, injector, and collector) quantum mechanically, only the $2 \mathrm{DEG}$ is quantum mechanically treated in Ref 19 . We believe that this is responsible for the difference.

In order to examine the connection between the electronic coherence and the nested peak structure, we first recall that in a single channel SFET, the conductance calculation $\frac{18}{}$, which takes into account the electronic coherence, results in

$$
G=\frac{e^{2}}{h} \frac{4 \cos ^{2} \frac{\phi_{\alpha}}{2} \sin ^{2}(K L)}{\sin ^{4} \frac{\phi_{\alpha}}{2}+4 \cos ^{2} \frac{\phi_{\alpha}}{2} \sin ^{2}(K L)},
$$

where $\phi_{\alpha} \equiv 2 m^{*} \alpha L / \hbar^{2}, K=\left[\left(k_{x}\right)_{i=1}+\left(k_{x}\right)_{i=2}\right] / 2$, and $\left(k_{x}\right)_{i=1}$ and $\left(k_{x}\right)_{i=2}(>0)$ are the two longitudinal wavevectors at $E_{F}$. The deviation of Eq. (11) from the sinusoidal behavior arises from the fact that the injector and collector behave as reflecting walls for the electron spin component antiparallel to the favored spin direction in the injector and collector. Thus the unfavored spin component may be reflected many times by the ideal injector and collector until it acquires, via the spin precession by the RSO coupling, some component parallel to the favored spin component and is transmitted to the injector or collector. Thus in addition to the direct contribution that does not undergo any reflection, there are $n$-th order contributions to the electron transmission that undergo the reflection $2 n$ times until being transmitted to the collector. In this sense, the coherent SFET is analogous (though not identical) to the Fabry-Perot interferometer. It is straightforward to verify that the amplitude of the $(n+1)$-th contribution is smaller than that of the $n$-th contribution by the factor $-\exp (i 2 K L) \sin ^{2}\left(\phi_{\alpha} / 2\right)$ for $n=0$ and by the factor $\exp (i 2 K L) \cos ^{2}\left(\phi_{\alpha} / 2\right)$ for $n \geq 1$. Here the zeroth $(n=0)$ contribution denotes the direct contribution. Note that the interference between the direct contribution and the multiply reflected contributions is destructive (constructive) when the phase $\exp (i 2 K L)$ is $+1(-1)$. This explains the factor $\sin ^{2}(K L)$ in Eq. (11), which predicts the suppression (enhancement) of the FWHM of the profile $G(\alpha)$ when $\sin ^{2}(K L) \approx 0(1)$. Since $K$ depends on $E_{F}$, this $K$ dependence also implies the $E_{F}$ dependence. To 
examine the coherence effects in a multichannel SFET, we use an intuitive generalization of Eq. (11) to a multichannel system;

$G=\frac{e^{2}}{h} \sum_{n=0}^{N_{\mathrm{ch}}-1} g_{n}=\frac{e^{2}}{h} \sum_{n=0}^{N_{\mathrm{ch}}-1} \frac{4 \cos ^{2} \frac{\phi_{\alpha}}{2} \sin ^{2}\left(K_{n} L\right)}{\sin ^{4} \frac{\phi_{\alpha}}{2}+4 \cos ^{2} \frac{\phi_{\alpha}}{2} \sin ^{2}\left(K_{n} L\right)}$,

where $K_{n} \equiv\left[\left(k_{x}\right)_{n, i=1}+\left(k_{x}\right)_{n, i=2}\right] / 2$, and $\left(k_{x}\right)_{n, i}(>0)$ is the longitudinal wavevector at $E_{F}$ for the subband $(n, i)$ (see Fig. 51). Equation (12) is expected to be a reasonable approximation when the inter-channel mixing is weak and each channel is close to an ideal 1D channel, which are indeed the case for $\alpha \lesssim 10 \times 10^{-12}$ $\mathrm{eVm}$ and $N_{\mathrm{ch}} \lesssim 10$ as demonstrated in Sec. [II In Fig. 7(b), the prediction of Eq. (12) (blue dashed line) for $G\left(\alpha^{*}\right)$ as a function of $E_{F}$ is compared with the numerical TB conductance result (red solid line), where $\alpha^{*}=3.94 \times 10^{-12} \mathrm{eVm}$ [vertical dashed line in Fig. 7(a)]. Since $\alpha^{*}$ is close to the position where the conductance retains its maximal value $\left(e^{2} / h\right) N_{\mathrm{ch}}$, the dips in $G\left(\alpha^{*}\right)$ imply the formation of the nested peak structure. To compare with the TB calculation result, $K_{n}$ and $L$ in Eq. (12) are expressed in terms of the TB parameters. To evaluate $K_{n}=\left[\left(k_{x}\right)_{n, i=1}+\left(k_{x}\right)_{n, i=2}\right] / 2$, we use the relation $E_{F}=2 t\left\{2-\cos \left[\left(k_{x}\right)_{n, i} a\right]-\cos [(n+1) \pi a / w]\right\}+$ $(-1)^{i}(\alpha / a) \sin \left[\left(k_{x}\right)_{n, i} a\right]-m^{*} \alpha^{2} / 2 \hbar^{2}$, which amounts to the TB approximation of Eq. (8). Here $w=a\left(N_{y}+1\right)$. To evaluate $L$, we use $L=a\left(N_{x}+1+\beta\right)$, where $\beta=0.6$ is an ad hoc fitting parameter much smaller than $N_{x}=749$. Note that Eq. (12) produces dips (blue dashed line) and moreover the dip positions predicted by Eq. (12) are in good agreement with the TB conductance calculation result (red solid line). Thus Eq. (12) reproduces the nested peak structure. We remark that when $\beta=0$ is used, the dips in the blue dashed line shift to the right by $0.0001 \sim 0.0003 t$ only (not shown), not affecting the agreement significantly.

The agreement provides a simple insight into the origin of the nested peak structure. A key observation is that while all $g_{n}$ 's in Eq. (12) produce the conductance peaks at the same $\alpha$, the peak widths may fluctuate considerably from channel to channel. The channel-to-channel peak width fluctuation arises from the dependence of the peak width on $K_{n}$. In particular when $\sin ^{2}\left(K_{n} L\right) \approx 0$, the peak produced by $g_{n}$ becomes very narrow. Thus when $\sin ^{2}\left(K_{n} L\right)$ is close to zero for one particular $n$, one very narrow peak for that particular $n$ is superposed with the other broad peaks, generating the nested peak structure. Figure 7 (c) plots $\sin ^{2}\left(K_{n} L\right)$ 's as a function of $E_{F}$. Note that whenever one of $\sin ^{2}\left(K_{n} L\right)$ vanishes, a dip appears in Fig. 7(b). We remark that though the nested peak structure is illustrated mainly for $N_{\mathrm{ch}}=3$, it appears for higher $N_{\text {ch }}$ as well. An example for $N_{\text {ch }}=20$ is visible in Fig. 2(a) near $\alpha=4.3 \times 10^{-12} \mathrm{eVm}$. For sufficiently large $N_{\mathrm{ch}}$, however, the nested peak structure is expected to vanish. The nested peak structure was not found in a two-dimensional coherent SFET $\underline{29}$, which amounts to the $N_{\mathrm{ch}} \rightarrow \infty$ limit.

The electronic coherence can give rise to another interesting effect when an external magnetic field $\vec{B}$ is applied, providing an additional source of the spin precession. Here we consider $\vec{B}=B_{\|} \hat{y}$ applied along the $y$-axis, so that the spin precession axis by $\vec{B}$ is parallel to that due to the RSO coupling in the ideal 1D situation. The spin precessions by $B_{\|}$and the RSO coupling differ in one crucial respect; When the electron reverses its motion, the direction of the spin precession by the RSO coupling is reversed while that of the spin precession by $B_{\|}$is not. For a single channel SFET, it is illustrated ${ }^{18}$ that this difference, combined with the coherent FabryPerot-like interference, gives rise to the splitting of the conductance peaks upon the application of $B_{\|}$. Figure 8 shows that the magnetic-field-induced peak splitting occurs also for multichannel SFETs. The numerical TB conductance calculation results are compared with the expression,

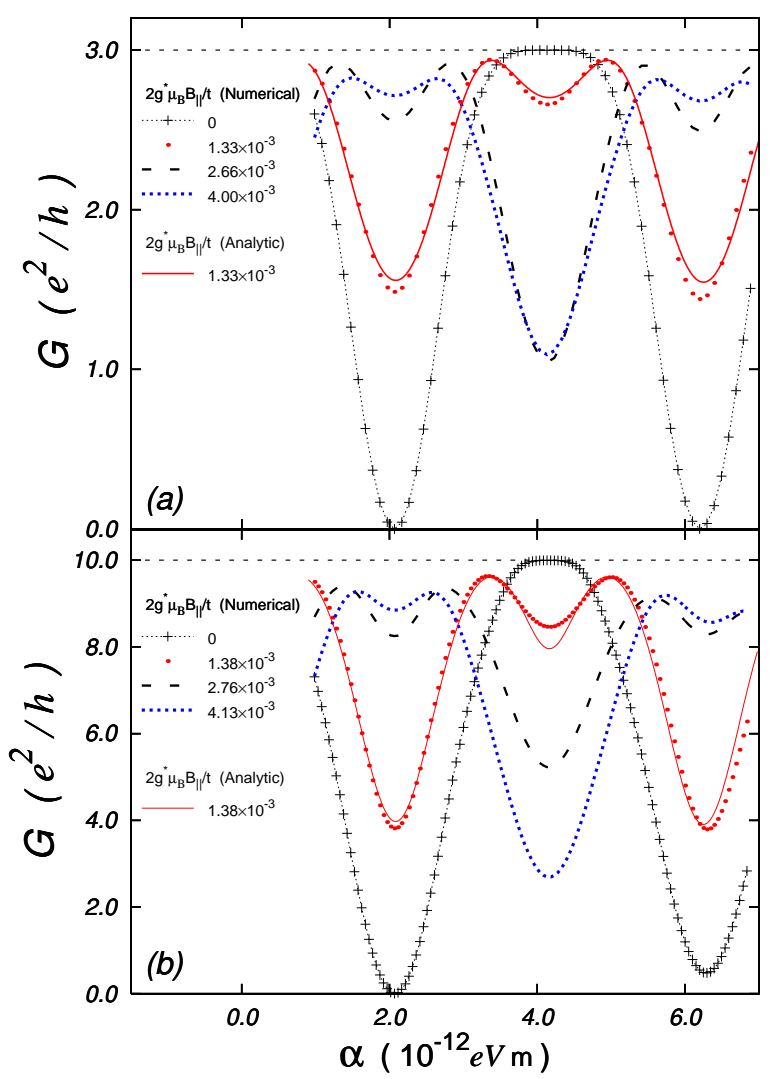

FIG. 8: (Color online) Conductance $G$ as a function of the RSO coupling parameter $\alpha$ for (a) $N_{\text {ch }}=3$ and (b) $N_{\mathrm{ch}}=10$. The solid lines in (a) and (b) show the analytic results obtained using Eq. (13) for a specific field strength $2 g^{*} \mu_{B} B_{\|} / t=1.33 \times 10^{-3}$ and $1.38 \times 10^{-3}$, respectively. Note that the analytic results are in good agreement with the numerical results. 


$$
G=\frac{e^{2}}{h} \sum_{n=0}^{N_{\mathrm{ch}}-1} g_{n}=\frac{e^{2}}{h} \sum_{n=0}^{N_{\mathrm{ch}}-1} \frac{4\left(\cos ^{2} \frac{\phi_{\alpha}}{2}-\sin ^{2} \frac{\phi_{B, n}}{2}\right) \sin ^{2} K_{n} L+4 \sin ^{2} \frac{\phi_{\alpha}}{2} \sin ^{2} \frac{\phi_{B, n}}{2}}{\left(\sin ^{2} \frac{\phi_{\alpha}}{2}+\sin ^{2} \frac{\phi_{B, n}}{2}\right)^{2}+4\left(\cos ^{2} \frac{\phi_{\alpha}}{2}-\sin ^{2} \frac{\phi_{B, n}}{2}\right) \sin ^{2} K_{n} L}
$$

which is an intuitive extension of the single channel formula in Ref $\stackrel{18}{\underline{18}}$. Here $\phi_{B, n}$ denotes the spin precession angle due to $B_{\|}$. For weak fields, it is given by $2 g^{*} \mu_{B} B_{\|} /\left(\hbar v_{F, n} / L\right)$, where $g^{*}$ is the effective Lande- $g$ factor, $\mu_{B}$ is the Bohr magneton, and $v_{F, n}=\hbar K_{n} / m^{*}$ is the group velocity of electrons with energy $E_{F}$ in the $n$-th channel ${ }^{30}$. Note that the predictions of Eq. (13) are in reasonable agreement with the numerical TB results both for $N_{\mathrm{ch}}=3$ and 10 .

\section{DISCUSSION}

An ideal spin injector/collector has been assumed in the preceding sections in order to focus on the multichannel effects. For a certain material to be an ideal spin injector/collector, (i) its spin polarization should be $100 \%$ and (ii) its effective mass and Fermi wavelength should match those for the 2DEG. While the condition (i) is satisfied in the so-called half-metals ${ }^{31}$ and the condition (ii) may be satisfied in ferromagnetic semiconductors or diluted magnetic semiconductors 32 , we are not aware of any material that satisfies the both conditions. In Sec. IVA, we thus address briefly effects of a nonideal spin injector/collector on the multichannel SFET.

\section{A. Nonideal spin injector/collector}

One of most explored and representative choices for a spin injector/collector is conventional metallic ferromagnets, whose spin polarization can be as high as $\sim 50 \%$. Use of conventional metallic ferromagnets requires a special care. Recent theories ${ }^{33}$ proposed that the spin injecton/detection rate can be greatly improved by introducing a thin insulator between a conventional ferromagnet and the 2DEG while without the insulator, the spin injection/detection rate was demonstrated $\underline{5}$ to be below $1 \%$ due to the so-called conductance mismatch problem ${ }^{6}$. The physics behind the insulator "prescription" for the conductance mismatch problem is that the tunneling through the insulating barrier becomes spindependent ${ }^{33}$ in the ferromagnet-insulator-2DEG geometry even though the insulator is nonmagnetic. Subsequent experiments ${ }^{34}$ using oxide insulating barriers have reported the enhanced spin polarization of $2-30 \%$ at room temperatures. Schottky tunnel barriers were also demonstrated to be effective and the spin polarization of 2-30\% has been reported 35 at low temperatures.

Here we calculate numerically the conductance of the multichannel SFET equipped with the nonideal spin in- jector/collector that consists of a conventional metallic ferromagnet-thin insulator-2DEG hybrid structure. One possible way to model the spin-dependent tunneling in the ferromagnet-insulator-2DEG structure is to explicitly take into account all three materials, ferromagnet, insulator, and 2DEG (Appendix E). This approach is however too demanding for numerical calculations since the Fermi wavelength in the ferromagnet is about two orders of magnitude smaller than that in the 2DEG. We thus adopt a simplified phenomenological description, where all effects of the ferromagnet (including not only its spin-dependent density of states but also the effective mass and the Fermi wavelength differences from those of the 2DEG) are taken into account by phenomenological spin-dependent hopping parameters for the tunneling through the insulator. To be specific, the TB Hamiltonian [Eq. (6)] for the coupling between the 2DEG and

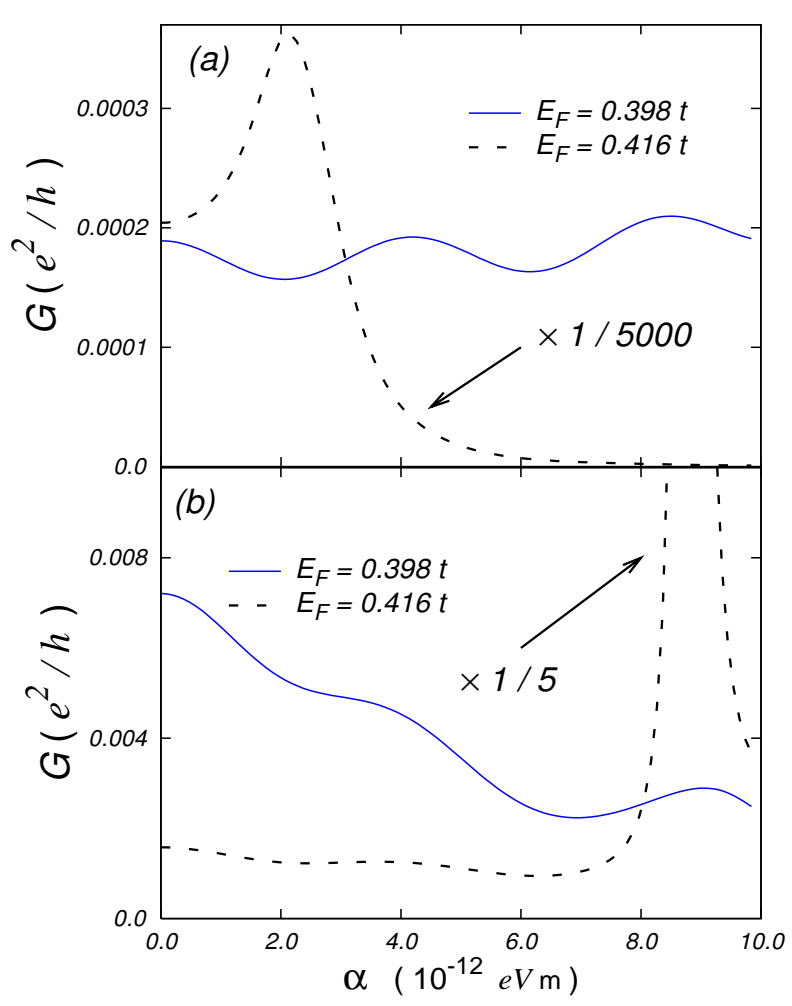

FIG. 9: (Color online) The conductance $G$ of a ballistic SFET with the nonideal injector/collector described by the modified $H_{\text {coupling }}^{\mathrm{TB}}\left[\right.$ Eq. (14)] and $H_{\mathrm{inj} / \mathrm{col}}^{\mathrm{TB}} \cdot N_{\mathrm{ch}}=3 \mathrm{in}$ (a) and $N_{\mathrm{ch}}=10$ in (b). The length $L$ of the 2DEG is $1.44 \mu \mathrm{m}$ in both (a) and (b). Other parameters are the same as those in Fig. 2] The spin polarization of the injected current for the ferromagnetinsulator-2DEG structure is assumed to be about $30 \%$. 


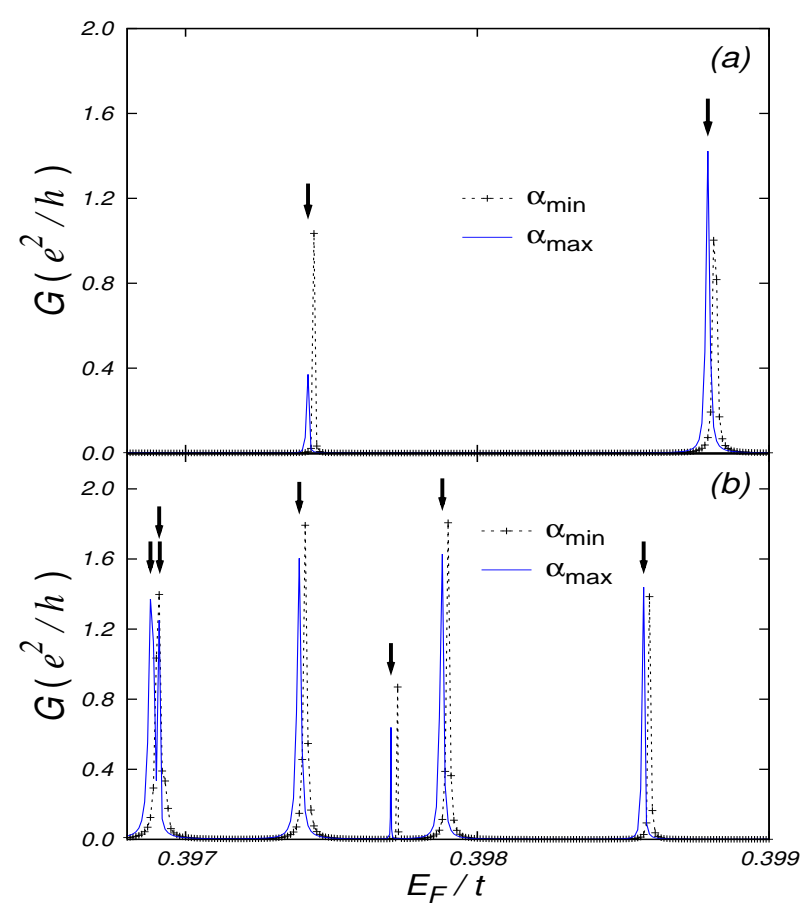

FIG. 10: (Color online) The conductance $G$ as a function of the Fermi energy $E_{F}$ with fixed $\alpha, \alpha_{\min } \equiv 2.07 \times 10^{-12}$ $\mathrm{eVm}$ and $\alpha_{\max } \equiv 4.13 \times 10^{-12} \mathrm{eVm}$, for $N_{\mathrm{ch}}=3$ in (a) and $N_{\mathrm{ch}}=10 \mathrm{in}$ (b). The peak positions of $G$ for $\alpha_{\max }$ are marked by arrows. Note that there are three closely spaced peaks near $E_{F}=0.397 t$. The length $L$ of the 2DEG is $1.44 \mu \mathrm{m}$.

the injector/collector is modified as follows:

$$
\begin{aligned}
H_{\text {coupling }}^{\mathrm{TB}}= & -\sum_{s_{x}} \sum_{j=1}^{N_{y}} t_{s_{x}}\left(c_{1, j, s_{x}}^{\dagger} c_{0, j, s_{x}}\right. \\
& \left.+c_{N_{x}+1, j, s_{x}}^{\dagger} c_{N_{x}, j, s_{x}}+\text { H.c. }\right),
\end{aligned}
$$

where $t_{s_{x}}$ is a spin-dependent hopping parameter that takes into account not only the spin-dependent tunneling through the insulator but also other effects from the effective mass and the Fermi wavelength differences between the ferromagnet and the 2DEG. Since all effects of the ferromagnet are already taken into account in $t_{s_{x}}$ [Eq. (14)], the injector/collector may be modelled as a nonmagnetic semiconducting lead, which can be described by modifying $H_{\mathrm{inj} / \mathrm{col}}^{\mathrm{TB}}$ [Eqs. (4) and (5)] so that similar contributions for $s_{x}=-1$ are also included. Although this description adopts a considerably simplified picture of the real system, it is still expected to capture the main physics of the spin injection/detection since, as demonstrated in Refs $\stackrel{33}{\underline{3}}$ and Appendix $\mathrm{E}$, the spin-dependent tunneling through the insulator is the main source of the sizable spin injection/detection rate $(\sim 30 \%)$ while the bulk properties of the ferromagnet generate much weaker contributions only. We choose the value of $t_{s_{x}}$ to be $0.1 t$ for $s_{x}=1$ and $0.073 t$ for $s_{x}=-1$, respectively. The resulting spin polarization of the injected current estimated
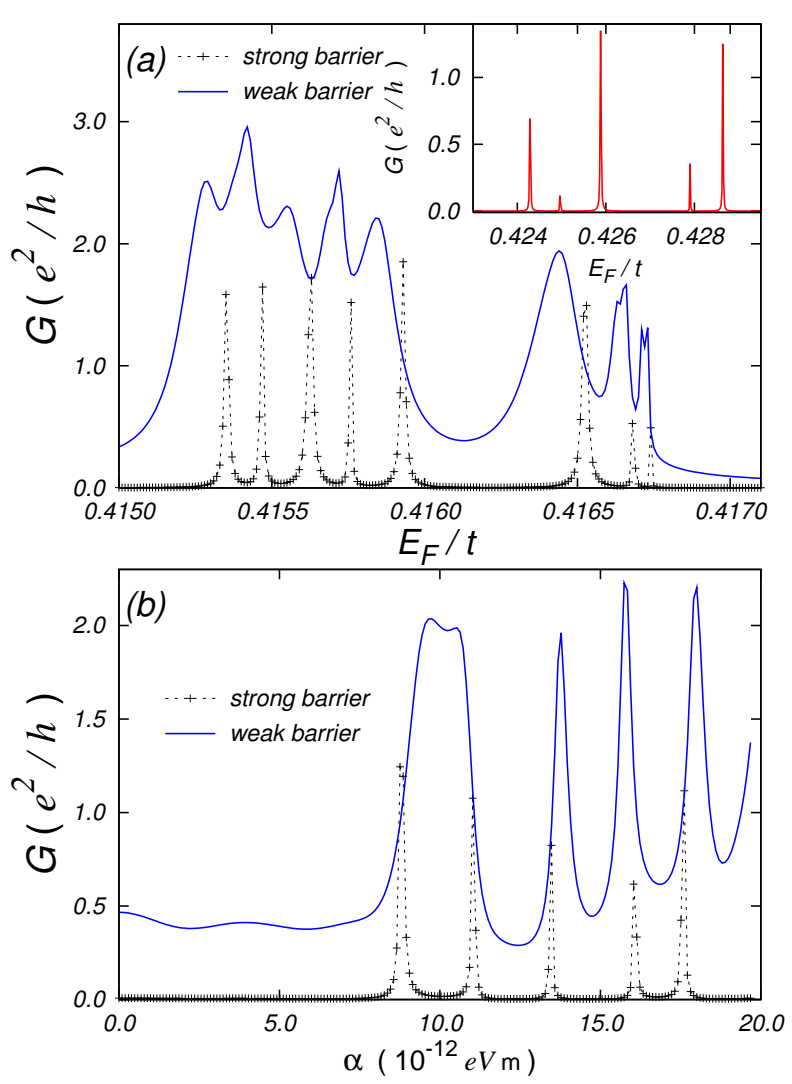

FIG. 11: (Color online) (a) The conductance $G$ as a function of $E_{F}$ for the SFET with two nonideal spin injector/collector that contains a thin insulating barrier. Here $N_{\mathrm{ch}}=10$ and $\alpha=8.3 \times 10^{-12} \mathrm{eVm}$. For the curves labelled as the strong and weak barriers, the hopping parameters [Eq. (14)] are $\left(t_{s_{x}=1}=\right.$ $\left.0.1 t, t_{s_{x}=-1}=0.073 t\right)$ and $\left(t_{s_{x}=1}=3 \times 0.1 t, t_{s_{x}=-1}=3 \times 0.073 t\right)$, respectively. Inset in (a) : $G\left(E_{F}\right)$ for $N_{\text {ch }}=3$. Note that the positions of the large resonance peaks nearly coincide with those of the nested peaks in Fig. [7(b). (b) The conductance $G$ as a function of $\alpha$ for the strong and weak barriers when $N_{\text {ch }}=10$ and $E_{F}=0.416 t$. The length $L$ of the 2DEG is $1.44 \mu \mathrm{m}$.

by $\left(t_{s_{x}=1}^{2}-t_{s_{x}=-1}^{2}\right) /\left(t_{s_{x}=1}^{2}+t_{s_{x}=-1}^{2}\right)$ is then $30 \%$, which is similar to the values reported in experiments 34 .

For $N_{\mathrm{ch}}=3$ and $E_{F}=0.398 t$ [solid line in Fig. 9(a)], $G$ clearly shows the modulation by $\alpha$ though the value of $G$ is considerably reduced below $0.001 \times\left(e^{2} / h\right)$ due to the insulating barriers and the ratio $G_{\max } / G_{\min } \simeq 1.7$ is also suppressed due to the reduced spin injection/detection rate of $30 \%$. Thus for this particular situation, $G$ shows rather expected behaviors. For other situations, somewhat unexpected behaviors also appear. For $N_{\mathrm{ch}}=3$ and $E_{F}=0.416 t$ [dashed line in Fig. [9(a)], the "periodic" modulation disappears and instead a large peak appears at $\alpha \simeq 2.1 \times 10^{-12} \mathrm{eVm}$ with the maximum conductance of $\sim 1.8 \times\left(e^{2} / h\right)$ (the peak height is scaled down by the factor of 5000 to fit in the vertical scale of the graph). For $N_{\mathrm{ch}}=10$ and $E_{F}=0.416 t$ [dashed line in Fig. 9(b)], a 


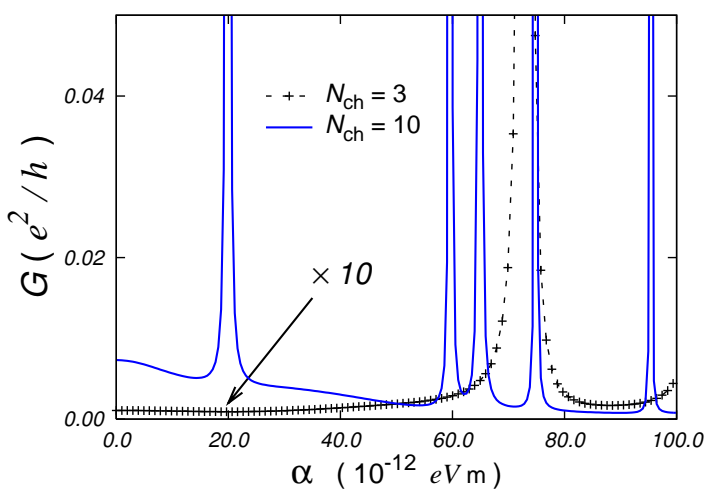

FIG. 12: (Color online) The conductance $G$ of a ballistic SFET with the nonideal injector/collector as a function of the RSO coupling parameter $\alpha$ at $T=0$. The length $L$ of the $2 \mathrm{DEG}$ is $0.1 \times 1.44 \mu \mathrm{m}$ and $E_{F}=0.398 t=0.103 \mathrm{eV}$.

large peak (near $\alpha \simeq 8.9 \times 10^{-12} \mathrm{eVm}$ ) coexists with the $G$ modulation in the lower $\alpha$ range. For $N_{\mathrm{ch}}=10$ and $E_{F}=0.398 t$ [solid line in Fig. 9(b)], the $G$ modulation is superimposed on a background slope.

To investigate how frequently such peaks appear, $G$ is evaluated as a function of $E_{F}$ for two representative values of $\alpha, \alpha_{\min } \equiv 2.07 \times 10^{-12} \mathrm{eVm}$ and $\alpha_{\max } \equiv$ $4.13 \times 10^{-12} \mathrm{eVm}$, which correspond to the positions of the local minimum and maximum of $G$ at $E_{F}=0.398 t$ for $N_{\mathrm{ch}}=3$. For $N_{\mathrm{ch}}=3$ [Fig. 10(a)], two peaks appear in the depicted $E_{F}$ range and for $N_{\mathrm{ch}}=10$ [Fig. 10(b)], seven peaks appear. Thus the occurrence of the peaks becomes more frequent with the increase of $N_{\mathrm{ch}}$. The calculation for $N_{\mathrm{ch}}=3,10$, and 20 over a much wider range of $E_{F}\left(0.360 t<E_{F}<0.440 t\right.$, not shown $)$ verifies this trend. Note that the peak positions for $\alpha_{\min }$ and $\alpha_{\max }$ are somewhat different, implying that the peak positions shift with $\alpha$. Considering that the peak heights $\left(\sim e^{2} / h\right)$ are three or four orders of magnitudes larger than the modulation amplitudes in Fig. 9, even tails of the peaks can be large "perturbations" to the modulation and thus the $\alpha$-dependence of the peak positions, though weak, can affect the modulation considerably. As remarked above, this perturbation appears more frequently for larger $N_{\mathrm{ch}}$.

To find the origin of the large peaks, their dependence on the strengths of the hopping parameters $t_{s_{x}}$ is examined. As demonstrated in Fig. 11, the peak widths for the large hopping parameters $\left(t_{s_{x}=1}=3 \times 0.1 t\right.$, $\left.t_{s_{x}=-1}=3 \times 0.073 t\right)$ are larger than those for the small hopping parameters $\left(t_{s_{x}=1}=0.1 t, t_{s_{x}=-1}=0.073 t\right)$. This difference in the peak widths implies that the large peaks are resonances due to the electron confinement effects of the insulators. Then at the energies where the resonances appear, the phase acquired by electrons during their motion from the injector to the collector should be an integer multiple of $\pi$. Interestingly this condition is the same as the condition for the occurrence of the nested peak structure in Sec. III The comparison between the inset in

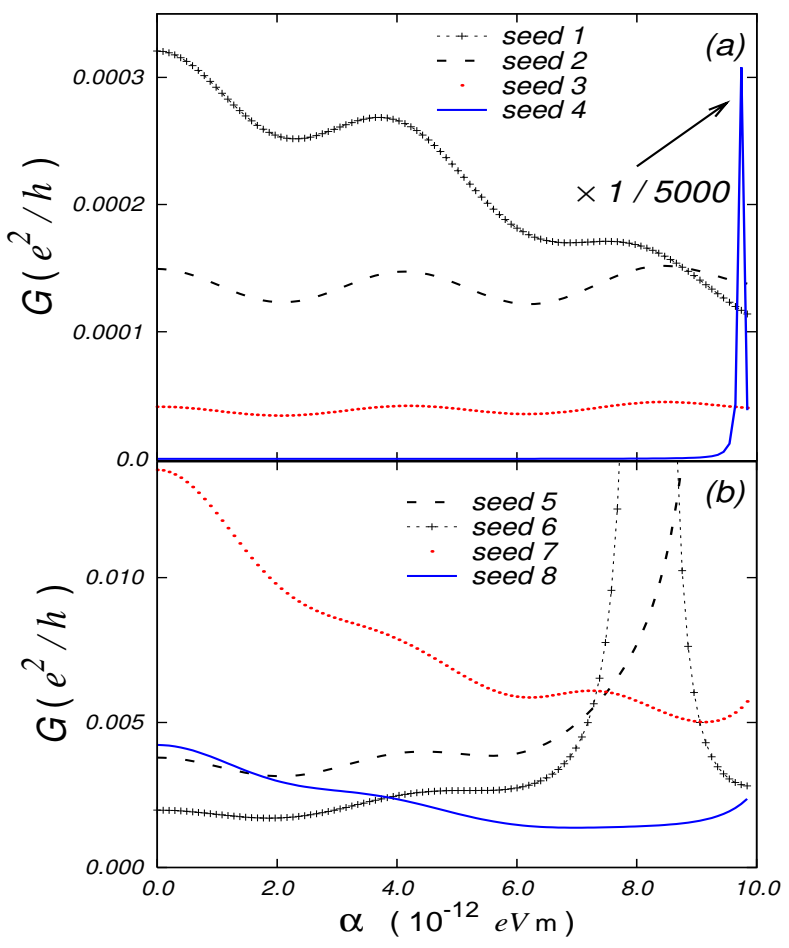

FIG. 13: (Color online) The conductance $G$ for several impurity-configurations in the 2DEG as a function of the RSO coupling parameter $\alpha$ for $N_{\mathrm{ch}}=3$ in (a) and $N_{\mathrm{ch}}=10$ in (b). The seed numbers represent different impurity configurations, all of which have the same mean free path $l=L / 3$. The other parameters are the same as in Fig. 9(a) and (b), respectively. The length $L$ of the 2DEG is $1.44 \mu \mathrm{m}$.

Fig. 11(a) for the large resonance peaks and Fig. 7(b) for the nested peaks indeed verifies this relation between the two phenomena. Recalling that the inter-channel coupling is weak for $N_{\mathrm{ch}} \lesssim 10$ and $\alpha \lesssim 10 \times 10^{-12} \mathrm{eVm}$, the correlation between the number of resonance peaks and $N_{\text {ch }}$ can also be explained easily since each channel separately gives rise to resonances.

Lastly we examine effects of the nonideal spin injector/collector on a shorter SFET. Figure 12 shows the conductance evolution for a SFET with relatively short $L=0.1 \times 1.44 \mu \mathrm{m}$ in a much wider window of $\alpha,(0 \sim$ $100) \times 10^{-12} \mathrm{eVm}$. Again large resonance peaks appear, distorting the spin-orbit-coupling-induced modulation of the conductance considerably. We remark that the decrease of $L$ clearly enhances the spacing of the resonances both in the $G$ vs. $\alpha$ plot and in the $G$ vs. $E_{F}$ plot (not shown). However considering that the decrease of $L$ also widens the minimum size of the $\alpha$ "window" for the observation of the spin-orbit-coupling-induced modulation of $G$ and that the change of $E_{F}$ simply "parallel" shifts the resonance positions in the $G$ vs. $\alpha$ plot, reducing $L$ does not appear to significantly facilitate the observation of the spin-orbit-coupling-induced modulation of $G$. 


\section{B. Scattering effects}

Another practical problem ignored in the preceding sections is the scattering effects. Scattering by impurities, which are isotropic, short-ranged, and spinindependent, can be modelled by using the TB Hamiltonian of the impurity potential given by $H_{\text {impurity }}^{\text {TB }}=$ $\sum_{s_{z}} \sum_{i=1}^{N_{x}} \sum_{j=1}^{N_{y}} U_{i, j} c_{i, j, s_{z}}^{\dagger} c_{i, j, s_{z}}$, where $U_{i, j}$ is a random variable whose value is uniformly distributed in the range $[-W, W]$. We investigate briefly the scattering effects in a weakly diffusive regime; $W$ is chosen in such a way that the mean free path $l$ is $L / 3$. The nonideal spin injector/collector is again assumed and $L=1.44 \mu \mathrm{m}$, $E_{F}=0.398 t$ are used. Figure 13 shows $G$ as a function of $\alpha$ for various realizations of the random potential $U_{i, j}$. For all realizations, $l$ is the same. Note that for certain realizations, the spin-orbit-coupling-induced modulation of $G$ is clearly visible while for other realizations, the modulations is strongly distorted by the resonance peaks. This difference is due to the fact that the positions of the resonances depend on details of the spatial profile of the random potential $U_{i, j}$. A similar result is obtained for a nonballistic single-channel SFET ${ }^{36}$. The results in Fig. 13 also imply that the conductance of a nonballistic multichannel SFET equipped with the nonideal spin injector/collector may show considerable sample-tosample fluctuations. Recalling that there are more resonance peaks for larger $N_{\mathrm{ch}}$, the sample-to-sample fluctuations are expected to be more significant for larger $N_{\mathrm{ch}}$. More systematic studies about nonballistic multichannel SFETs with the nonideal injector/collector are necessary.

\section{CONCLUSION}

The issue of multichannel effects were addressed. Via the numerical conductance calculation using the tightbinding approximation, it has been demonstrated that the conductance modulation becomes less ideal with the increase of the channel number $N_{\mathrm{ch}}$. For the ideal spin injector/collector, the conductance modulation ratio $G_{\max } / G_{\min }$ as a function of $N_{\text {ch }}$ (from a few to 90) has been examined for the RSO coupling parameter $\alpha \sim 5 \times 10^{-12} \mathrm{eVm}$ and for the larger $\alpha \sim 50 \times 10^{-12}$ $\mathrm{eVm}$. It has been found that the decay of the modulation ratio with $N_{\mathrm{ch}}$ is considerably faster for the larger $\alpha$. Through the study of the energy dispersion relations and the spin configurations of eigenstates, it has been revealed that with the increase of $N_{\mathrm{ch}}$ and $\alpha$, the spin configuration deviates further from the ideal $1 \mathrm{D}$ configuration due to the formation of the phase-slip-like structure and the gradual drift of the spin angle, and that the energy dispersion deviates further from the ideal quadratic dispersion since the avoided crossing distorts the dispersion in a wider range of the longitudinal wavevectors. It turns out that those deviations are rather small when $2 m^{*} \alpha w / \hbar^{2} \ll 2 \pi$ while they become significant when
$2 m^{*} \alpha w / \hbar^{2} \gtrsim 2 \pi$. Thus this result provides insights to the regime $2 m^{*} \alpha w / \hbar^{2} \gtrsim 2 \pi$, where the inter-channel coupling is expected to be significant ${ }^{4,19}$. Effects of the electronic coherence in the multichannel regime were also examined and we have found the nested peak structure, which was attributed to the coherent Fabry-Perot-like interference in the multichannel regime. We have also verified that the magnetic field-induced peak splitting, reported previously 18 for a single-channel SFET, persists in the multichannel regime. For the nonideal spin injector/collector that consists of a conventional metallic ferromagnet-thin insulator-2DEG hybrid structure, it has been found that large resonance peaks due to the electron confinement by the insulators may strongly distort the spin-orbit-coupling-induced modulation of the conductance. When the 2DEG becomes weakly diffusive, the conductance modulation signal shows considerable sample-to-sample fluctuations.

\section{Acknowledgments}

We acknowledge H.-S. Sim, Hyowon Park, Woojoo Sim, and J.-H. Kim for their help on numerical calculations. This work was supported by the SRC/ERC program (Grant No. R11-2000-071) and the Basis Research Program (Grant No. R01-2005-000-10352-0) of MOST/KOSEF, by the POSTECH Core Research Program, and by the Korea Research Foundation Grant (Grant No. KRF-2005-070-C00055 and BK21 program) funded by the Korean Government (MOEHRD).

\section{Appendix A: Ideal injector and collector}

As a test of the ideal spin injector, we calculate the electron transmission probability $T_{\text {interface }}$ through the injector-2DEG interface for an electron incident from the injector side with the incident momentum $\mathbf{k}=\left(k_{x}, k_{y}\right)$ and its spin pointing along the favored direction, $x$-axis. The model Hamiltonian of the injector-2DEG hybrid system reads $H_{\mathrm{hyb}}=\vartheta(x) H_{2 \mathrm{D}}+\vartheta(-x) H_{\mathrm{inj}}$, where $\vartheta(x)$ is the Heaviside step function. Since we are mainly interested in interface effects on the spin injection, the confinement potential $V_{\mathrm{c}}$ in $H_{2 \mathrm{D}}$ and $H_{\text {inj }}$ is ignored below. For $\alpha=0, T_{\text {interface }}$ is 1 since $H_{2 \mathrm{D}}$ and $H_{\mathrm{inj}}$ are identical for the electron with its spin pointing along the $x$-axis. For nonzero $\alpha, T_{\text {interface }}$ will deviate from one. The evaluation of $T_{\text {interface }}$ is straightforward. Recalling that the eigenspin direction in the 2DEG differs from $(+x)$-direction and that the injected electron wave is thus a superposition of two plane waves with different spin directions, one needs to match properly the superposition with the electron state in the injector. The result is shown in Fig. 14 for the three incident angles $\theta=0$, $\pi / 6$, and $\pi / 4$, where $\theta \equiv \sin ^{-1}\left(k_{y} /|\mathbf{k}|\right)$. Note that re-

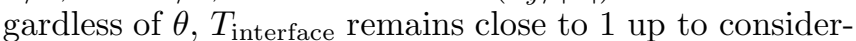
ably large $\alpha, 100 \times 10^{-12} \mathrm{eVm}$. Note also that $T_{1}$ and $T_{2}$ 
$\left(T_{1}+T_{2}=T_{\text {interface }}\right)$, transmission probabilities into each of the two plane wave states in the $2 \mathrm{DEG}$, remain close to their values $\cos ^{2}(\theta / 2+\pi / 4)$ and $\sin ^{2}(\theta / 2+\pi / 4)$ in the limit $\alpha \rightarrow 0$, indicating that the spin of the injected electron points along the $x$-direction right after the injection. Figure 14 thus verifies the ideal spin injector. The ideal spin collector can be verified in a similar way.

\section{Appendix B: Symmetry analysis}

Symmetries often facilitate the analysis of a system and provide useful information ${ }^{37}$. Since $H_{2 \mathrm{D}}$ [Eq. (1)] is invariant under the translation along the $x$-axis, an energy eigenfunction can be written as $\Psi(x, y)=$ $e^{i k_{x} x}\left[\psi_{\uparrow}(y) \chi_{\uparrow}+\psi_{\downarrow}(y) \chi_{\downarrow}\right]$, where $\psi_{\uparrow, \downarrow}(y)$ is the spin component along the $( \pm z)$-direction, $\chi_{\uparrow} \doteq\left(\begin{array}{l}1 \\ 0\end{array}\right)$ Pauli spin-up state and $\chi_{\downarrow} \doteq\left(\begin{array}{c}0 \\ 1\end{array}\right)$ spin-down state.

$H_{2 \mathrm{D}}$ also commutes with two other symmetry operators $\Theta$ and $\Pi_{x} \mathcal{D}_{\text {spin }}(\hat{\mathbf{x}}, \pi)$, where $\Theta$ is the time-reversal operator, $\Pi_{x}$ is the mirror reflection operator with respect to the $y z$-plane in the orbital space, and $\mathcal{D}_{\text {spin }}(\hat{\mathbf{x}}, \pi)$ is the rotation operator with respect to the $x$-axis by the angle $\pi$ in the spin space. To examine implications of these symmetry operators, it is convenient to use the representation $\mathcal{D}_{\text {spin }}(\hat{\mathbf{x}}, \pi)=\exp \left(-i \pi S_{x} / \hbar\right) \doteq-i \sigma_{x}$, and $\Theta \doteq \sigma_{y} K$, where $K$ is the complex-conjugation operator in the real space representation with the $z$-axis as the spin quantization axis. Application of $\Theta \Pi_{x} \mathcal{D}_{\text {spin }}(\hat{\mathbf{x}}, \pi)$ on $\Psi(x, y)$ results in $e^{i k_{x} x}\left[\psi_{\uparrow}^{*}(y) \chi_{\uparrow}-\psi_{\downarrow}^{*}(y) \chi_{\downarrow}\right]$. Since $\left[H_{2 \mathrm{D}}, \Theta \Pi_{x} \mathcal{D}_{\text {spin }}(\hat{\mathbf{x}}, \pi)\right]=0$, the resulting state should also be an energy eigenfunction with the energy eigenvalue same as that for $\Psi(x, y)$. Then by comparing $\Psi(x, y)$ with $\Theta \Pi_{x} \mathcal{D}_{\text {spin }}(\hat{\mathbf{x}}, \pi) \Psi(x, y)$, one finds that without loss of generality, $\psi_{\uparrow}(y)$ may be chosen to be real and $\psi_{\downarrow}(y)$ may be chosen to be imaginary. Recalling the spinor representation $\left(\begin{array}{c}\cos \theta / 2 \\ e^{i \phi} \sin \theta / 2\end{array}\right)$ for the spin with the polar angle $\theta$ and the azimuthal angle $\phi$, one finds that $\phi$

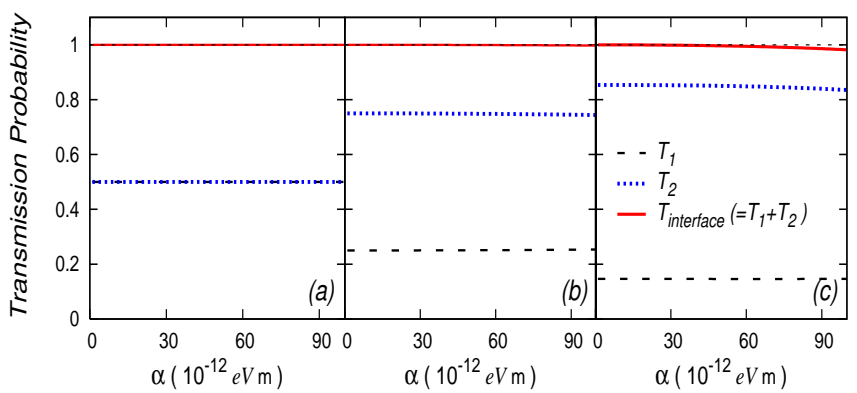

FIG. 14: (Color online) Transmission probability $T_{\text {interface }}$ through the injector-2DEG interface as a function of the RSO coupling parameter $\alpha$. The electron is injected from the injector side with its spin pointing along the $(+x)$-direction and with the incident angle $\theta$ ( 0 for normal incidence). (a) $\theta=0$, (b) $\theta=\pi / 6$ and (c) $\theta=\pi / 4 . T_{1}$ and $T_{2}\left(T_{1}+T_{2}=T_{\text {interface }}\right)$ are the transmission probabilities into the two involved eigenstates in the 2DEG. for the energy eigenfunctions is $\pm \pi / 2$ and that the spin direction of the energy eigenfunctions always lies within the $y z$-plane.

When the confining potential is symmetric $V_{\mathrm{c}}(y)=$ $V_{\mathrm{c}}(w-y)$, which is the case for the hard wall confinement potential, $H_{2 \mathrm{D}}$ allows another symmetry operation $i \Pi_{y} \mathcal{D}_{\text {spin }}(\hat{\mathbf{y}}, \pi)$, where $\Pi_{y}$ is the mirror reflection operator with respect to the $y=w / 2$ plane in the orbital space and $\mathcal{D}_{\text {spin }}(\hat{\mathbf{y}}, \pi)$ is the rotation operator with respect to the $y$-axis by the angle $\pi$ in the spin space. Since $\left[i \Pi_{y} \mathcal{D}_{\text {spin }}(\hat{\mathbf{y}}, \pi)\right]^{2}=I$, possible eigenvalues of $i \Pi_{y} \mathcal{D}_{\text {spin }}(\hat{\mathbf{y}}, \pi)$ are +1 and -1 , and the energy eigenfunctions can be classified into even and odd parity states accordingly. For notational convenience, we choose $\phi=+\pi / 2$ for even parity states and $\phi=-\pi / 2$ for odd parity states. At the special point $y=w / 2, \Pi_{y}$ plays no role and the parity becomes identical to the eigenvalue of $i \mathcal{D}_{\text {spin }}(\hat{\mathbf{y}}, \pi)=\sigma_{y}$. Thus at $y=w / 2$, the spin of the even parity states points towards $(+y)$-direction $(\theta=\pi / 2$, $\phi=+\pi / 2)$ and the spin of odd parity states points towards $(-y)$-direction $(\theta=\pi / 2, \phi=-\pi / 2)$. For $y \neq w / 2$, the spin in general deviates from $( \pm y)$-direction. But due to the parity constraint, the spin directions at $y$ and $w-y$ are correlated; $\theta(y)+\theta(w-y)=\pi$.

\section{Appendix C: Hard wall confinement : Exact results}

An exact energy eigenstate $\Psi(x, y)=e^{i k_{x} x}\left[\psi_{\uparrow}(y) \chi_{\uparrow}+\right.$ $\left.\psi_{\downarrow}(y) \chi_{\downarrow}\right]$ of $H_{2 \mathrm{D}}$ [Eq. (1)] with energy $E$ can be expressed as a linear superposition of plane waves as follows,

$$
\Psi(x, y)=\sum_{j=1}^{4} c_{j} \Psi_{\mathbf{k}_{j}}(x, y)
$$

where the plane wave $\Psi_{\mathbf{k}_{j}}(x, y)=e^{i \mathbf{k}_{j} \cdot \mathbf{r}} u_{j}\left(\mathbf{k}_{j}\right)$ is an energy eigenfunction of $H_{2}$ in the absence of the confinement potential $\left[V_{c}(y)=0\right], \mathbf{r}=(x, y), \mathbf{k}_{j}=\left(k_{x}, k_{y, j}\right)$, and $k_{y, j}$ 's are four solutions of $E=\hbar^{2}\left(k_{x}^{2}+k_{y, j}^{2}\right) / 2 m^{*}+$ $(-1)^{j}|\alpha|\left(k_{x}^{2}+k_{y, j}^{2}\right)^{1 / 2}$ (see Fig. 4). When all four solutions are real (denoted by the blue solid line in Fig. 4), one may take $k_{y, 1}=-k_{y, 3}$ and $k_{y, 2}=-k_{y, 4}$. But in certain situations (denoted by the red dashed line in Fig. 4), only two solutions are real and the other two remaining solutions are pure imaginary. We remark that in order to obtain a complete energy spectrum, one should consider such situations as well. In such situations, one may take $k_{y, 1}=-k_{y, 3}$ for the two real solutions and $k_{y, 2}^{*}=-k_{y, 4}$ for the other two purely imaginary solutions. The procedure described below applies to both situations (blue solid line and red dashed line) denoted in Fig. 4. For the spinors $u_{1}\left(\mathbf{k}_{1}\right)$ and $u_{2}\left(\mathbf{k}_{2}\right)$, we choose the representation,

$$
u_{1}\left(\mathbf{k}_{1}\right)=\frac{1}{\sqrt{2}}\left(\begin{array}{c}
1 \\
i \frac{\alpha}{|\alpha|} \frac{k_{x}+i k_{y, 1}}{\left(k_{x}^{2}+k_{y, 1}^{2}\right)^{1 / 2}}
\end{array}\right) \text {, }
$$


and

$$
u_{2}\left(\mathbf{k}_{2}\right)=\frac{1}{\sqrt{2}}\left(\begin{array}{c}
1 \\
-i \frac{\alpha}{|\alpha|} \frac{k_{x}+i k_{y, 2}}{\left(k_{x}^{2}+k_{y, 2}^{2}\right)^{1 / 2}}
\end{array}\right)
$$

For the spinors $u_{3}\left(\mathbf{k}_{3}\right)$ and $u_{4}\left(\mathbf{k}_{4}\right)$, we choose their representation in such a way that the plane waves $\Psi_{\mathbf{k}_{j}}(x, y)$ 's satisfy the relations, $\Psi_{\mathbf{k}_{3}}(x, y)=i \Pi_{y} \mathcal{D}_{\text {spin }}(\hat{\mathbf{y}}, \pi) \Psi_{\mathbf{k}_{1}}(x, y)$ and $\Psi_{\mathbf{k}_{4}}(x, y)=i \Pi_{y} \mathcal{D}_{\text {spin }}(\hat{\mathbf{y}}, \pi) \Psi_{\mathbf{k}_{2}}(x, y)$, which is possible since $i \Pi_{y} \mathcal{D}_{\text {spin }}(\hat{\mathbf{y}}, \pi)$ is a symmetry operation of the system.

The four coefficients $c_{j}$ 's in Eq. (C1) are fixed by the hard-wall boundary conditions, $\Psi(x, y=0)=\Psi(x, y=$ $w)=0$, which lead to a secular equation for a $4 \times 4$ matrix. Solving this equation is facilitated by the symmetry operation $i \Pi_{y} \mathcal{D}_{\text {spin }}(\hat{\mathbf{y}}, \pi)$. Since $i \Pi_{y} \mathcal{D}_{\text {spin }}(\hat{\mathbf{y}}, \pi)$ has only two eigenvalues, +1 and -1 , the eigenstates can be classified into even and odd parity states. For an even parity state, one may choose $c_{3}=c_{1}$ and $c_{4}=c_{2}$, and for an odd parity state, one may choose $c_{3}=-c_{1}$ and $c_{4}=-c_{2}$. Thus the secular equation for the $4 \times 4$ matrix is blockdiagonalized into two $2 \times 2$ matrices, one for even parity states and the other for odd parity states. By solving the two $2 \times 2$ matrices separately, one obtains the eigenenergy $E$ and the corresponding eigenstate $\Psi(x, y)$ for even and odd parity states. Figure 5 shows the energy dispersion for various combinations of $\alpha$ and $w$. Figure 6 shows the spin profiles of selected eigenstates.

\section{Appendix D: Perturbation theory}

Although the exact eigen wavefunctions and energydispersion relations are readily available, perturbation theory calculations are still useful in order to gain insights into the $\alpha$-dependence of eigen transport channels. Below we treat the RSO coupling as a perturbation and examine its effects on eigen transport channels. Since the longitudinal momentum $k_{x}$ is an exact quantum number of $H_{2 \mathrm{D}}$ in Eq. (11), one may work with the reduced Hamiltonian $H_{2 \mathrm{D}}\left(k_{x}\right)=H_{0}+H_{R}$ that acts on the transverse part $\psi(y)$ of the original wavefunction $\Psi(x, y)=e^{i k_{x} x} \psi(y)$. Here the unperturbed Hamiltonian $H_{0}$ and the perturbation $H_{R}$ are given by

$$
H_{0} \equiv\left[\frac{\hbar^{2} k_{x}^{2}}{2 m^{*}}+\frac{p_{y}^{2}}{2 m^{*}}+V_{\mathrm{c}}(y)\right] I, \quad H_{R} \equiv \alpha\left(\sigma_{x} \frac{p_{y}}{\hbar}-\sigma_{y} k_{x}\right),
$$

where $I$ is the identity operator acting on spin.

In the zeroth order in $\alpha$, each energy eigenvalue of $H_{2 \mathrm{D}}\left(k_{x}\right)$ is doubly degenerate with $E_{n, 1}^{(0)}=E_{n, 2}^{(0)}=$ $\hbar^{2} k_{x}^{2} / 2 m^{*}+\mathcal{E}_{n}$, where $\mathcal{E}_{n} \equiv \hbar^{2} k_{n, y}^{2} / 2 m^{*}$ is the energy of the $n$-th $(n=0,1,2, \cdots)$ excitation mode in the transverse motion and $k_{n, y} \equiv(n+1) \pi / w$. The corresponding eigenfunctions are $\psi_{n, 1}^{(0)}(y)=\phi_{n}(y) \cdot\left(\chi_{\uparrow}+i \chi_{\downarrow}\right) / \sqrt{2}$ and $\psi_{n, 2}^{(0)}(y)=\phi_{n}(y) \cdot\left(\chi_{\uparrow}-i \chi_{\downarrow}\right) / \sqrt{2}$ respectively, where $\phi_{n}(y) \equiv \sqrt{2 / w} \sin k_{n, y} y$, and $\chi_{\uparrow}=\left(\begin{array}{l}1 \\ 0\end{array}\right), \chi_{\downarrow}=\left(\begin{array}{l}0 \\ 1\end{array}\right)$ are the spinors pointing along the $( \pm z)$-directions.

Corrections to the eigenenergies can be obtained easily. The first order corrections to $E_{n, i}^{(0)}(i=1,2)$ are given by the representation of $H_{R}$ within the two-dimensional degenerate subspace:

$$
\left(\begin{array}{cc}
\left\langle\psi_{n, 1}^{(0)}\left|H_{R}\right| \psi_{n, 1}^{(0)}\right\rangle & \left\langle\psi_{n, 1}^{(0)}\left|H_{R}\right| \psi_{n, 2}^{(0)}\right\rangle \\
\left\langle\psi_{n, 2}^{(0)}\left|H_{R}\right| \psi_{n, 1}^{(0)}\right\rangle & \left\langle\psi_{n, 2}^{(0)}\left|H_{R}\right| \psi_{n, 2}^{(0)}\right\rangle
\end{array}\right)=\left(\begin{array}{cc}
-\alpha k_{x} & 0 \\
0 & \alpha k_{x}
\end{array}\right) .
$$

Thus one obtains the first order energy correction $E_{n, i}^{(1)}=$ $(-1)^{i} \alpha k_{x}$. Note that since the $2 \times 2$ matrix of Eq. (D2) is diagonal, $\psi_{n, 1}^{(0)}$ and $\psi_{n, 2}^{(0)}$ are the proper zeroth order eigenfunctions in view of the degenerate perturbation theory. The second order energy corrections are given by

$$
E_{n, i}^{(2)}=\left\langle\psi_{n, i}^{(0)}\left|H_{R} \frac{\mathcal{P}_{n}}{E_{n, i}^{(0)}-H_{0}} H_{R}\right| \psi_{n, i}^{(0)}\right\rangle,
$$

where the projection operator $\mathcal{P}_{n}$ is defined by $\mathcal{P}_{n} \equiv 1-$ $\sum_{i=1}^{2}\left|\psi_{n, i}^{(0)}\right\rangle\left\langle\psi_{n, i}^{(0)}\right|$. By using the identity $\mathcal{P}_{n} \sigma_{y}\left|\psi_{n, i}^{(0)}\right\rangle=0$ and the relation $\left[H_{0}, \sigma_{x}\right]=0$, Eq. (D3) can be written as

$$
E_{n, i}^{(2)}=\frac{\alpha^{2}}{\hbar^{2}} \sum_{l \neq n} \frac{1}{\mathcal{E}_{n}-\mathcal{E}_{l}}\left|\int d y \phi_{l}^{*}(y) p_{y} \phi_{n}(y)\right|^{2},
$$

which reduces further to

$$
E_{n, i}^{(2)}=-\frac{m^{*} \alpha^{2}}{2 \hbar^{2}}
$$

with the help of the identities $p_{y}=\left(m^{*} / i \hbar\right)\left[y, H_{0}\right]$ and $\sum_{l} \phi_{l}^{*}\left(y^{\prime}\right) \phi_{l}(y)=\delta\left(y-y^{\prime}\right)$. Thus up to the second order in $\alpha$, the energy dispersion relation is given by

$$
E_{n, i}=\frac{\hbar^{2} k_{x}^{2}}{2 m^{*}}+\mathcal{E}_{n}+(-1)^{i} \alpha k_{x}-\frac{m^{*} \alpha^{2}}{2 \hbar^{2}} .
$$

The result is shown in Fig. 5. Here we remark that although Eq. (D6) is derived for the hard-wall confinement potential, the first- and second-order corrections to the eigenenergy are independent of $V_{\mathrm{c}}(y)$; its change alters only the zeroth order contribution $\mathcal{E}_{n}$. A similar result for a harmonic confinement has been reported previously 38 .

Next we examine corrections to energy eigenstates. According to the degenerate perturbation theory, the first order corrections $\left|\psi_{n, i}^{(1)}\right\rangle=\mathcal{P}_{n}\left|\psi_{n, i}^{(1)}\right\rangle+\mathcal{Q}_{n}\left|\psi_{n, i}^{(1)}\right\rangle$, where $\mathcal{Q}_{n} \equiv \sum_{i=1}^{2}\left|\psi_{n, i}^{(0)}\right\rangle\left\langle\psi_{n, i}^{(0)}\right|$ and $\mathcal{P}_{n}+\mathcal{Q}_{n}=1$, are given by

$$
\mathcal{P}_{n}\left|\psi_{n, i}^{(1)}\right\rangle=\sum_{m \neq n} \sum_{j=1}^{2}\left|\psi_{m, j}^{(0)}\right\rangle \frac{1}{E_{n, i}^{(0)}-E_{m, j}^{(0)}}\left\langle\psi_{m, j}^{(0)}\left|H_{R}\right| \psi_{n, i}^{(0)}\right\rangle,
$$

and

$$
\mathcal{Q}_{n}\left|\psi_{n, i}^{(1)}\right\rangle=\left|\psi_{n, \bar{i}}^{(0)}\right\rangle \frac{1}{E_{n, i}^{(1)}-E_{n, \bar{i}}^{(1)}}\left\langle\psi_{n, \bar{i}}^{(0)}\left|H_{R} \frac{P_{n}}{E_{n}^{(0)}-H_{0}} H_{R}\right| \psi_{n, i}^{(0)}\right\rangle,
$$


where $\bar{i}=2$ for $i=1$ and $\bar{i}=1$ for $i=2$. Both Eqs. (D7) and (D8) can be considerably simplified. With the help of the identities $\mathcal{P}_{n} \sigma_{y}\left|\psi_{n, i}^{(0)}\right\rangle=0, p_{y}=\left(m^{*} / i \hbar\right)\left[y, H_{0}\right]$, and $\sum_{l} \phi_{l}^{*}\left(y^{\prime}\right) \phi_{l}(y)=\delta\left(y-y^{\prime}\right)$, one finds

$$
\begin{gathered}
\mathcal{P}_{n}\left|\psi_{n, i}^{(1)}\right\rangle=(-1)^{i+1} \frac{m^{*} \alpha}{\hbar^{2}}\left(y-\langle y\rangle_{n}\right)\left|\psi_{n, \bar{i}}^{(0)}\right\rangle, \\
\mathcal{Q}_{n}\left|\psi_{n, i}^{(1)}\right\rangle=0,
\end{gathered}
$$

where $\langle y\rangle_{n} \equiv \int d y \phi_{n}^{*}(y) y \phi_{n}(y)=w / 2$. Then up to the first order in $\alpha$, the eigen wavefunctions are given by

$$
\psi_{n, i}(y)=\psi_{n, i}^{(0)}(y)+(-1)^{i+1} \frac{m^{*} \alpha\left(y-\langle y\rangle_{n}\right)}{\hbar^{2}} \psi_{n, \bar{i}}^{(0)}(y),
$$

We remark that although Eq. (D11) is derived for the hard wall confinement, it holds for other confinement potentials $V_{\mathrm{c}}(y)$ as well when $\langle y\rangle_{n}$ is evaluated for the new $V_{\mathrm{c}}(y)$. The second order correction $\left|\psi_{n, i}^{(2)}\right\rangle=\mathcal{P}_{n}\left|\psi_{n, i}^{(2)}\right\rangle+$ $\mathcal{Q}_{n}\left|\psi_{n, i}^{(2)}\right\rangle$ is given by

$$
\begin{aligned}
& \mathcal{P}_{n}\left|\psi_{n, i}^{(2)}\right\rangle= \frac{\mathcal{P}_{n}}{E_{n, i}^{(0)}-H_{0}}\left(H_{R}-E_{n, i}^{(1)}\right) \mathcal{Q}_{n}\left|\psi_{n, i}^{(1)}\right\rangle \\
&+\left[\frac{\mathcal{P}_{n}}{E_{n, i}^{(0)}-H_{0}}\left(H_{R}-E_{n, i}^{(1)}\right)\right]^{2}\left|\psi_{n, i}^{(0)}\right\rangle, \\
& \mathcal{Q}_{n}\left|\psi_{n, i}^{(2)}\right\rangle=\left|\psi_{n, \bar{i}}^{(2)}\right\rangle \frac{1}{E_{n, i}^{(1)}-E_{n, \bar{i}}^{(1)}}\left(\left\langle\psi_{n, i}^{(0)}\left|H_{R} \mathcal{P}_{n}\right| \psi_{n, i}^{(2)}\right\rangle\right. \\
& \\
&\left.-\frac{E_{n, i}^{(2)}}{E_{n, i}^{(1)}-E_{n, \bar{i}}^{(1)}}\left\langle\psi_{n, \bar{i}}^{(0)}\left|H_{R} \mathcal{P}_{n}\right| \psi_{n, i}^{(1)}\right\rangle\right) .
\end{aligned}
$$

With the help of similar identities, one finds

$$
\begin{gathered}
\mathcal{P}_{n}\left|\psi_{n, i}^{(2)}\right\rangle=\left(\frac{m^{*} \alpha w}{\hbar^{2}}\right)^{2}\left[2 \sum_{n^{\prime} \neq n} \frac{\hbar^{2} / m^{*} w^{2}}{E_{n, i}^{(0)}-E_{n^{\prime}, \bar{i}}^{(0)}}\left|\psi_{n^{\prime}, \bar{i}}^{(0)}\right\rangle\left(k_{x}\langle y\rangle_{n^{\prime}, n}\right)\right. \\
\left.-\sum_{n^{\prime}, n^{\prime \prime} \neq n} \frac{E_{n^{\prime}, i}^{(0)}-E_{n^{\prime \prime}, i}^{(0)}}{E_{n, i}^{(0)}-E_{n^{\prime \prime}, i}^{(0)}}\left|\psi_{n^{\prime \prime}, i}^{(0)}\right\rangle \frac{\langle y\rangle_{n^{\prime \prime}, n^{\prime}}}{w} \frac{\langle y\rangle_{n^{\prime}, n}}{w}\right],(\mathrm{D} 14) \\
\mathcal{Q}_{n}\left|\psi_{n, i}^{(2)}\right\rangle=0
\end{gathered}
$$

where $\langle y\rangle_{n, n^{\prime}} \equiv\left\langle\phi_{n}|y| \phi_{n^{\prime}}\right\rangle$. We remark that these expressions for the second correction are again independent of the choice of $V_{\mathrm{c}}(y)$.

These expressions contain the information on the local spin direction. Since the Hamiltonian $H_{2 \mathrm{D}}$ is invariant under the transformation $\Theta \Pi_{x} \mathcal{D}_{\text {spin }}(\hat{\mathbf{x}}, \pi)$, the local spin direction is always perpendicular to the $x$-axis as demonstrated in Appendix B and it can be parameterized by a single angle $\theta_{n, i}(y)$ within the $y z$ plane; $\psi_{n, i}(y)=\left|\psi_{n, i}(y)\right| \cdot\left(\cos \frac{\theta_{n, i}(y)}{2} \chi_{\uparrow}+i \sin \frac{\theta_{n, i}(y)}{2} \chi_{\downarrow}\right)$. The local spin direction is pointing $-y,+z,+y$, and $-z$ axis when $\theta_{n, i}$ is $-\pi / 2,0, \pi / 2$, and $\pm \pi$, respectively. By using the perturbatively obtained wavefunctions, the angle $\theta_{n, i}(y)$ can be expanded as a power series of $\alpha$, $\theta_{n, i}=\theta_{n, i}^{(0)}+\theta_{n, i}^{(1)}+\theta_{n, i}^{(2)}+\cdots$, where the zeroth and the first order contributions are

$$
\theta_{n, 1}^{(0)}(y)=\frac{\pi}{2}, \quad \theta_{n, 1}^{(1)}(y)=-\frac{2 m^{*} \alpha}{\hbar^{2}}\left(y-\langle y\rangle_{n}\right),
$$

and

$$
\theta_{n, 2}^{(0)}(y)=-\frac{\pi}{2}, \quad \theta_{n, 2}^{(1)}(y)=-\frac{2 m^{*} \alpha}{\hbar^{2}}\left(y-\langle y\rangle_{n}\right) .
$$

A similar result has been reported previously 25 . Note that the first order contributions change linearly with $y$, explaining the gradual drift in Fig. 6. Note also that despite the $y$-dependence of $\theta_{n, i}$ the local spin directions of $\psi_{n, 1}(y)$ and $\psi_{n, 2}(y)$ remain antiparallel to each other $\left[\psi_{n, 1}^{\dagger}(y) \psi_{n, 2}(y)=0\right]$ at every position $y$ up to the first order in $\alpha$ since $\left|\theta_{n, 1}(y)-\theta_{n, 2}(y)\right|=\pi$ up to the first order in $\alpha$. The second order contribution $\theta_{n, i}^{(2)}(y)$ can be also obtained (expressions not shown) from the perturbative expressions for $\psi_{n, i}(y)$ and it turns out that when $\theta_{n, i}^{(2)}(y)$ 's are taken into account, the local spin directions of $\psi_{n, 1}(y)$ and $\psi_{n, 2}(y)$ are not antiparallel to each other any more. Solid lines in Fig. [6 show $\theta_{n, i}(y)$ calculated from the perturbative expressions for $\psi_{n, i}(y)$, where it is clear that the deviation of $\left|\theta_{n, 1}(y)-\theta_{n, 2}(y)\right|$ from $\pi$ becomes more evident for larger $\alpha$. The second order contribution $\theta_{n, i}^{(2)}(y)$ is responsible for the phase-slip-like structure in Fig. 6.

\section{Appendix E: A nonideal spin injector}

Here we consider the spin injection from a nonideal injector, which consists of a conventional metallic ferromagnet(F)-insulator(I)-2DEG heterostructure. The differences of the effective masses, the densities of states, and the Fermi wavelengths between the $\mathrm{F}$ and the 2DEG are taken into account and the electron transport within the $\mathrm{F}$ and the 2DEG is assumed to be ballistic. It has

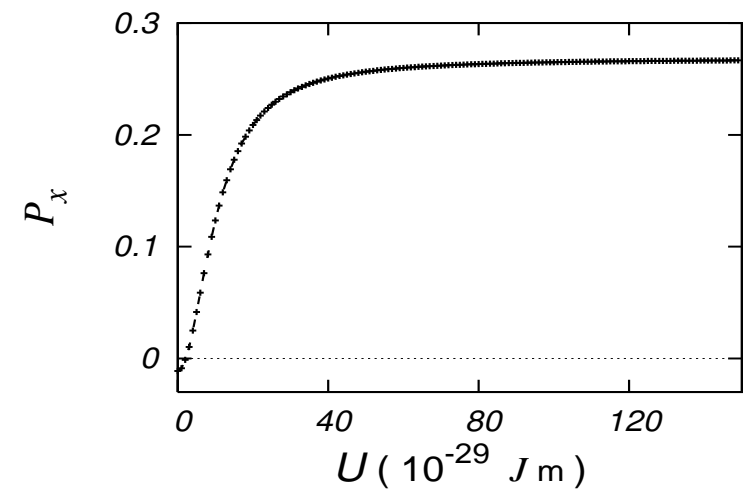

FIG. 15: Spin polarization of injected currents, $P_{x}$ whose spin is along the $(+x)$-direction, as a function of the tunnel contact parameter $U$. 
been reported ${ }^{39}$ that the detailed structure of the spin dependent density of states within the insulator can influence the spin polarization of the injected electron. In this paper, however, we ignore such details for simplicity and model the insulator by a $\delta$-function potential $U \delta(x)$, where $U$ denotes the tunnel contact parameter ${ }^{40,41}$. The model Hamiltonian of the F-I-2DEG hybrid system reads $H_{\text {hyb }}^{\prime}=\vartheta(x) H_{2 \mathrm{D}}+\vartheta(-x) H_{\mathrm{F}}+U \delta(x)$, where $H_{\mathrm{F}}$ has the form $H_{\mathrm{F}}=\mathbf{p}^{2} / 2 m_{\text {electron }}-h_{0} \sigma_{x}+V_{0}$. Here, $h_{0}$ represents the exchange interaction of the Stoner model in the $\mathrm{F}$, and $V_{0}$ denotes the difference between the bottoms of the energy bands for the $\mathrm{F}$ and 2DEG. Below we ignore the confinement potential $V_{c}$ and focus on the case of the normal incidence $(\theta=0)$ from the $\mathrm{F}$ since the Fermi wave vector in the $\mathrm{F}$ is much larger than that of the $2 \mathrm{DEG}^{29}$ and thus the normally incident electrons constitute a major portion of the injected electrons. We choose the value of the Fermi energy and the exchange interaction $h_{0}$ of the $\mathrm{F}$ to be $3.500 \mathrm{eV}$ and $1.750 \mathrm{eV}$, respectively, and set the Fermi energy
$E_{F}$ of the 2DEG to $E_{F}=0.398 t=0.103 \mathrm{eV}$. Thus $V_{0}=(0.103-3.500) \mathrm{eV}$. Figure 15$]$ shows the spin polarization $P_{x}$ of the injected currents as a function of the tunnel contact parameter $U$, where $P_{x}$ is a measure of the spin polarization along the $(+x)$-direction and is defined by $\left(T_{\text {interface }}^{s_{x}=1}-T_{\text {interface }}^{s_{x}=-1}\right) /\left(T_{\text {interface }}^{s_{x}=1}+T_{\text {interface }}^{s_{x}=-1}\right)$. Here $T_{\text {interface }}^{s_{x}}$ represents the electron transmission probability through the F-I-2DEG structure for an incident electron with the spin $s_{x}$. Note that while $P_{x}$ is only a few percent in the absence of $U$, it grows close to $30 \%$. This demonstrates clearly that the spin-dependent tunneling over the insulator barrier is the main source of the spin polarization of the injected current while the bulk properties of the $\mathrm{F}$ are rather irrelevant factors for the spin polarization of the injected current. This observation, which is in agreement with Refs $\stackrel{33.41}{4}$, justifies the simplified description in Sec. IVA of the F-I-2DEG structure, where the differences between the two bulks ( $\mathrm{F}$ and 2DEG) are ignored while the spin-dependent tunneling over the $\mathrm{I}$ is taken into account.
* Electronic address: jsjeong@postech.ac.kr

1 Yu. A. Bychkov and E. I. Rashba, J. Phys. C 17, 6039 (1984); JETP Lett. 39, 78 (1984).

2 J. Nitta, T. Akazaki, H. Takayanagi, and T. Enoki, Phys. Rev. Lett. 78, 1335 (1997); T. Koga, J. Nitta, T. Akazaki, and H. Takayanagi, Phys. Rev. Lett. 89, 046801 (2002).

${ }^{3}$ For a review, see I. Žutic̀, J. Fabian and S. Das Sarma, Rev. Mod. Phys. 76, 323 (2004).

${ }^{4}$ S. Datta and B. Das, Appl. Phys. Lett. 56, 665 (1990).

5 A. T. Filip, B. H. Hoving, F. J. Jedema, B. J. van Wees, B. Dutta, and S. Borghs, Phys. Rev. B 62, 9996 (2000).

${ }^{6}$ G. Schmidt, D. Ferrand, L. W. Molenkamp, A. T. Filip, and B. J. van Wees, Phys. Rev. B 62, R4790 (2000).

7 A. Bournel, P. Dollfus, S. Galdin, F.-X. Musalem and P. Hesto, Solid State Commun. 104, 85 (1997); A. Bournel, P. Dollfus, P. Bruno, and P. Hesto, Eur. Phys. J. AP 4, 1 (1998);

8 A. A. Kiselev and K. W. Kim, Phys. Rev. B 61, 13115 (2000); Phys. Status Solidi (b) 221, 491 (2000).

9 M. Cahay and S. Bandyopadhyay, Phys. Rev. B 69, 045303 (2004); A. G. Mal'shukov and K. A. Chao, Phys. Rev. B 61, R2413 (2000); S. Pramanik, S. Bandyopadhyay, and M. Cahay, Phys. Rev. B 68, 075313 (2003); Appl. Phys. Lett. 84, 266 (2004); J. Schliemann, J. C. Egues, and D. Loss, Phys. Rev. Lett. 90, 146801 (2003); E. Shafir, M. Shen, and S. Saikin, Phys. Rev. B 70, R241302 (2004); B. K. Nikolić and S. Souma, Phys. Rev. B 71, 195328 (2005).

10 S. Bandyopadhyay and M. Cahay, Appl. Phys. Lett. 85, 1433 (2004); S. Pramanik, S. Bandyopadhyay, and M. Cahay, IEEE Trans. Nanotechnol. 4, 2 (2005).

11 J. Luo, H. Munekata, F. F. Fang, and P. J. Stiles, Phys. Rev. B 41, 7685 (1990).

12 G. Engels, J. Lange, Th. Schäpers, and H. Lüth, Phys. Rev. B 55, R1958 (1997).

13 J. P. Heida, B. J. van Wees, J. J. Kuipers, T. M. Klapwijk, and G. Borghs, Phys. Rev. B 57, 11911 (1998).

14 M. Schultz, F. Heinrichs, U. Merkt, T. Colin, T. Skauli, and S. Lovold, Semicond. Sci. Technol. 11, 1168 (1996); J. Hong, J. Lee, S. Joo, K. Rhie, B. C. Lee, J. Lee, S. An, J. Kim, and K.-H. Shin, J. Korean Phys. Soc. 45, 197 (2004).

15 Th. Schäpers, J. Nitta, H. B. Heersche, and H. Takayanagi, Phys. Rev. B 64, 125314 (2001).

16 F. Mireles and G. Kirczenow, Europhys. Lett. 59, 107 (2002); Phys. Rev. B 66, 214415 (2002).

17 M. H. Larsen, A. M. Lunde, and K. Flensberg, Phys. Rev. B 66, 033304 (2002).

18 H.-W. Lee, S. Çalışkan and H. Park, Phys. Rev. B 72, 153305 (2005).

19 F. Mireles and G. Kirczenow, Phys. Rev. B 64, 024426 (2001).

20 S. Datta, Electronic transport in mesoscopic systems (Cambridge University Press, Cambridge, 1995).

21 See for instance Eq. (1) in T. P. Pareek and P. Bruno, Phys. Rev. B 65, 241305(R) (2002).

22 M. P. Anantram and T. R. Govindan, Phys. Rev. B 58, 4882 (1998).

23 E. A. de Andrada e Silva and G. C. La Rocca, Phys. Rev. B 67, 165318 (2003).

24 A. V. Moroz and C. H. W. Barnes, Phys. Rev. B 60, 14272 (1999); ibid. 61, R2464 (2000); M. Governale and U. Zülicke, Phys. Rev. B 66, 073311 (2002).

25 W. Häusler, Phys. Rev. B 63, 121310(R) (2001).

26 J. C. Egues, G. Burkard, and D. Loss, Appl. Phys. Lett. 82, 2658 (2003).

27 P. Šeba, P. Exner, K. N. Pichugin, A. Vyhnal, and P. Středa, Phys. Rev. Lett. 86, 1598 (2001).

${ }^{28}$ For a single-channel SFET, it has been demonstrated ${ }^{18}$ that the $E_{F}$-dependence of the conductance profile is averaged out at temperatures $k_{B} T$ sufficiently larger than $\pi \hbar v_{F} / L$, where $v_{F}$ is the Fermi velocity, and the high temperature expression is given by $G(\alpha)=$ $\left(2 e^{2} / h\right) \cos ^{2}\left(\phi_{\alpha} / 2\right) /\left[1+\cos ^{2}\left(\phi_{\alpha} / 2\right)\right]$. Then a trivial generalization to a multichannel SFET is obtained by multiplying $N_{\text {ch }}$ to the single-channel result. 
29 T. Matsuyama, C.-M. Hu, D. Grundler, G. Meier, and U. Merkt, Phys. Rev. B 65, 155322 (2002).

${ }^{30}$ In the evaluation of $v_{F, n}$, smaller correction due to the $\mathrm{RSO}$ coupling is ignored. In the presence of $B_{\|}$, the definition of $K_{n}$ is altered to $K_{n}=\left[\left(k_{x}^{\text {right }}\right)_{n, i=1}+\left(k_{x}^{\text {right }}\right)_{n, i=2}+\right.$ $\left.\left|\left(k_{x}^{\text {left }}\right)_{n, i=1}\right|+\left|\left(k_{x}^{\text {left }}\right)_{n, i=2}\right|\right] / 4$, where $\left(k_{x}^{\text {right }}\right)_{n, i}(>0)$ and $\left(k_{x}^{\text {left }}\right)_{n, i}(<0)$ are the longitudinal wavevectors at $E_{F}$ for the right and left moving electrons. In the absence of $B_{\|}$, this definition reduces to the definition right below Eq. (12).

31 R. A. de Groot, F. M. Mueller, P. G. van Engen, and K. H. J. Buschow, Phys. Rev. Lett. 50, 2024 (1983); K. P. Kämper, W. Schmitt, and G. Güntherodt, R. J. Gambino, and R. Ruf, Phys. Rev. Lett. 59, 2788 (1987); J. -H. Park, E. Vescovo, H. -J. Kim, C. Kwon, R. Ramesh, and T. Venkatesan, Nature (London) 392, 794 (1998).

32 H. Ohno, A. Shen, F. Matsukura, A. Oiwa, A. Endo, S. Katsumoto, and Y. Iye, Appl. Phys. Lett. 69, 363 (1996); H. Ohno, Science 281, 951 (1998); H. Ohno, J. Magn. Magn. Mater. 200, 110 (1999).

33 E. I. Rashba, Phys. Rev. B 62, R16267 (2000); D. L. Smith and R. N. Silver, Phys. Rev. B 64, 045323 (2001); A. Fert and H. Jaffres, Phys. Rev. B 64, 184420 (2001).
34 V. F. Motsnyi, P. Van Dorpe, W. Van Roy, E. Goovaerts, V. I. Safarov, G. Borghs, and J. De Boeck, Phys. Rev. B 68, 245319 (2003); X. Jiang, R. Wang, R. M. Shelby, R. M. Macfarlane, S. R. Bank, J. S. Harris, and S. S. P. Parkin, Phys. Rev. Lett. 94, 056601 (2005).

${ }^{35}$ H. J. Zhu, M. Ramsteiner, H. Kostial, M. Wassermeier, H. -P. Schönherr, and K. H. Ploog, Phys. Rev. Lett. 87, 016601 (2001); A. T. Hanbicki, O. M. J. van 't Erve, R. Magno, G. Kioseoglou, C. H. Li, B. T. Jonker, G. Itskos, R. Mallory, M. Yasar, and A. Petrou, Appl. Phys. Lett. 82, 4092 (2003).

36 M. Cahay and S. Bandyopadhyay, cond-mat/0306283

37 F. Zhai and H. Q. Xu, Phys. Rev. Lett. 94, 246601 (2005).

38 L. Serra, D. Sánchez, and R. López, Phys. Rev. B 72, 235309 (2005).

39 J. M. De Teresa, A. Barthélémy, A. Fert, J. P. Contour, R. Lyonnet, F. Montaigne, P. Seneor, and A. Vaurès, Phys. Rev. Lett. 82, 4288 (1999).

40 G. E. Blonder, M. Tinkham, and T. M. Klapwijk, Phys. Rev. B 25, 4515 (1982).

41 C.-M. Hu and T. Matsuyama, Phys. Rev. Lett. 87, 066803 (2001). 